\title{
Definizione di banche dati e procedure per la valorizzazione del Palazzo Centrale dell'Università di Pavia
}

\author{
Francesca Picchio \\ Elisabetta Doria \\ Alessia Miceli
}

Abstract

Nel dibattito contemporaneo che riguarda le pratiche per la conservazione del patrimonio costruito, la coscienza del valore che un bene architettonico ricopre nella memoria collettiva diventa la base per sviluppare una metodologia di indagine volta sia alla conservazione dei suoi elementi architettonici sia, per diretta conseguenza, alla sua valorizzazione. Questo è ancor più vero nel caso di architetture che abbiano sviluppato un profondo e simbiotico legame col proprio contesto urbano e territoriale, come il Palazzo Centrale dell'Università di Pavia. L'evoluzione dell'edificato che, durante i secoli, ha visto la rifunzionalizzazione e il conseguente rimaneggiamento di molti spazi, ha portato ad una varietà architettonica che si manifesta sia a scala dell'edificio, con differenti caratteristiche compositive ed una molteplicità di apparati decorativi, sia a scala urbana, attraverso una conformazione planimetrica che si apre e si fonde con la città, diventando essa stessa parte del tessuto storico. II progetto di documentazione di questo eterogeneo sistema di spazi tra loro concatenati, gestito dal laboratorio di ricerca Dada Lab dell'Università degli Studi di Pavia a partire dal 2019, garantisce la restituzione di disegni e modelli 3D; un corpus documentale delle facciate dei cortili e dei fronti strada del Palazzo. Descrivere accuratamente l'aspetto dei vari ambienti, delle superfici murarie e il loro stato di conservazione è funzionale alla pianificazione dei processi di manutenzione programmata dell'edificio nell'obiettivo di strutturare un sistema che possa promuovere e trasmettere nel tempo il carattere identitario di città universitaria.

Parole chiave

documentazione digitale, conservazione, virtualità.
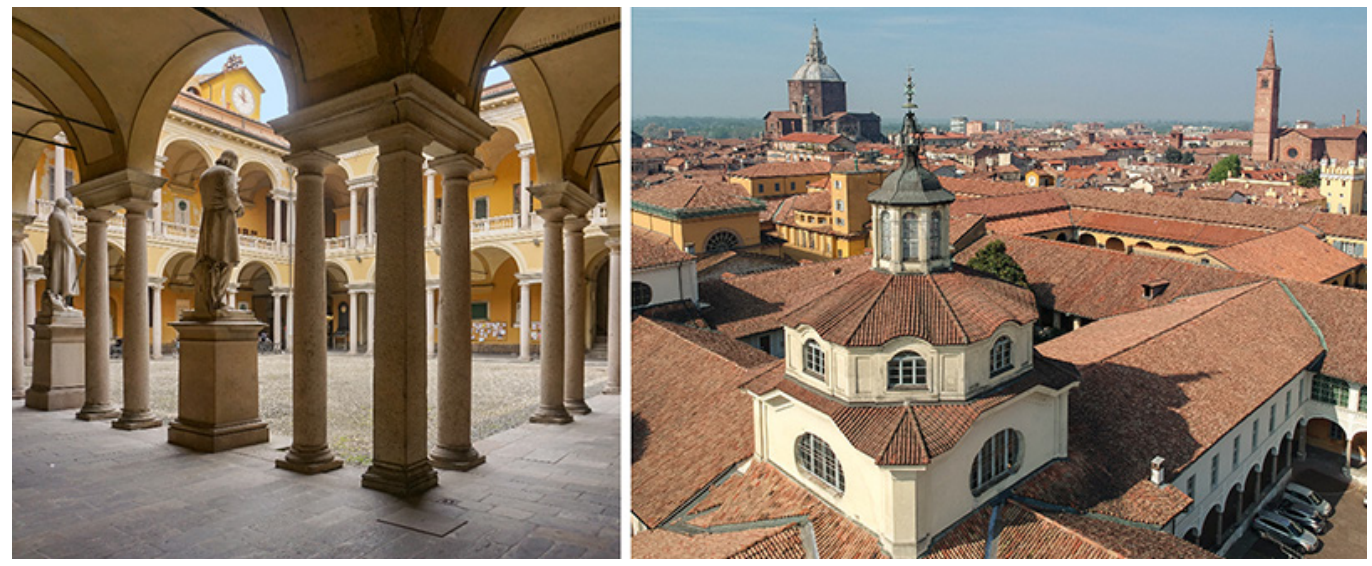


\section{Intoduzione: memoria e documentazione per la salvaguardia del bene architettonico}

La consapevolezza [I] che la finalità delle operazioni di documentazione non sia più soltanto la conservazione del bene ma anche la sua valorizzazione nell'aspetto immateriale [2] - ovvero nella capacità di configurarsi come elemento identificativo per la città - diventa essenziale per la strutturazione di una serie di interventi volti alla salvaguardia e alla gestione consapevole del bene architettonico [3].

II valore che la collettività attribuisce al patrimonio costruito deriva sia dal suo ruolo nelle vicende storiche che lo hanno interessato che, in misura non inferiore, da come viene percepito allinterno di uno specifico contesto, aspetto condizionato dalle variazioni di utilizzo del bene o dalle trasformazioni subite dal tessuto storico che ne hanno modificato le relazioni spaziali. La fruizione dello spazio urbano o architettonico diviene pericolosamente minata quando si esplicitano livelli di alterazione dello stato di conservazione del bene che ne compromettono l'usabilità, sottraendo loro la coltivazione ed il mantenimento dei valori legati ai caratteri identitari e alla loro preservazione nel tempo [4]. Per gli attori coinvolti nei processi di trasformazione sul patrimonio costruito, queste considerazioni diventano fonte di una consapevolezza che reclama, come punto di partenza, un'attenta conoscenza dell'oggetto e, nel caso di complessi particolarmente estesi, un'analisi sia del bene come elemento architettonico che come parte del sistema urbano. La consapevolezza, intesa come "strategia" sia in termini di conoscenza condivisa, sia in termini di responsabilità d'azione, diventa elemento guida del processo di documentazione come strumento di preservazione della memoria [5].

Le attività di documentazione del Palazzo Centrale dell'Università di Pavia, promosso dall'Ufficio Tecnico dell'Università e coordinato dal laboratorio di ricerca Dada Lab dell'Università degli Studi di Pavia, se da un lato puntano, attraverso il disegno, a restituire graficamente quegli aspetti legati alla conservazione delle superfici architettoniche [6], dall'altro cercano di sfruttare quanto messo in campo dalle tecnologie e dai sistemi di fruizione virtuale dello spazio per caratterizzare il database digitale e popolarlo di quelle informazioni che incrementano la conoscenza del bene, amplificando, attraverso la discretizzazione e la selezione delle informazioni, i valori materiali ed immateriali appartenenti al sistema architettonico.

Fig. I. Immagini del Palazzo dell'Università di Pavia, che mostrano il suo rapporto con il tessuto del centro storico.
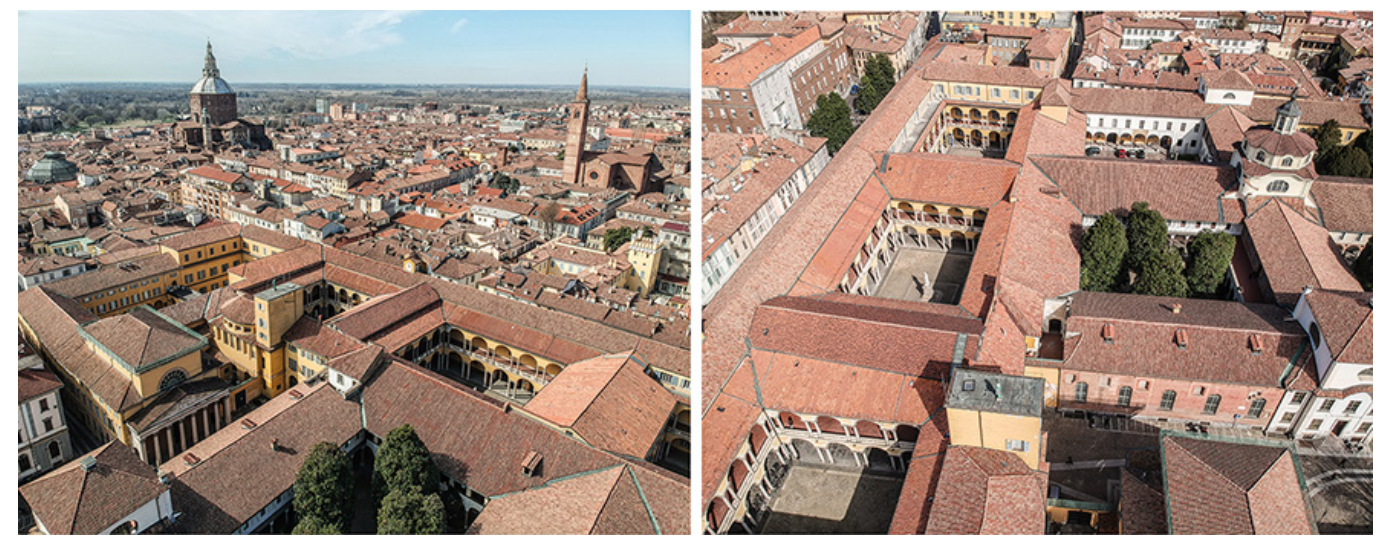

\section{La complessa relazione tra Città e Università}

L'antico patrimonio universitario italiano è storicamente caratterizzato da un forte legame con il tessuto urbano. Una delle ragioni di questo rapporto è la sua caratteristica di attingere a differenti tipi di patrimonio costruito come risorse per la propria espansione territoriale, quali la cessione di beni privati, ecclesiastici o di utilità pubblica. In questo senso si potrebbe affermare che le università italiane sono tra i primi fautori di interventi di riuso del patrimonio costruito per fini diversi da quelli originari [7]. L'Università di Pavia, una delle più antiche 
Fig. 2.I fronti del complesso architettonico dell'Università si configurano come fronti urbani, riprendendo i caratteri compositivi dello spazio col quale dialogano. L'esterno di Corso Strada Nuova appare lineare e compatto e racchiude i cortili interni che si configurano come ambienti concatenati ma fortemente diversificati.
Fig. 3. Le planimetrie storiche della città d Pavia illustrano alcune fas significative dell'espansiosignificative dell'Università. Nella prima (I 640) I'Università prima (1640) I'Università
di Pavia è profondamente innestata nel reticolato urbano. Nella second ( I 823) l'Università è riconoscibile e si sviluppa attorno ai 4 cortili, anche se ancora non è avvenuta l'annessione dell'ex ospedale San Matteo (Archivio dei Musei Civici di Pavia).
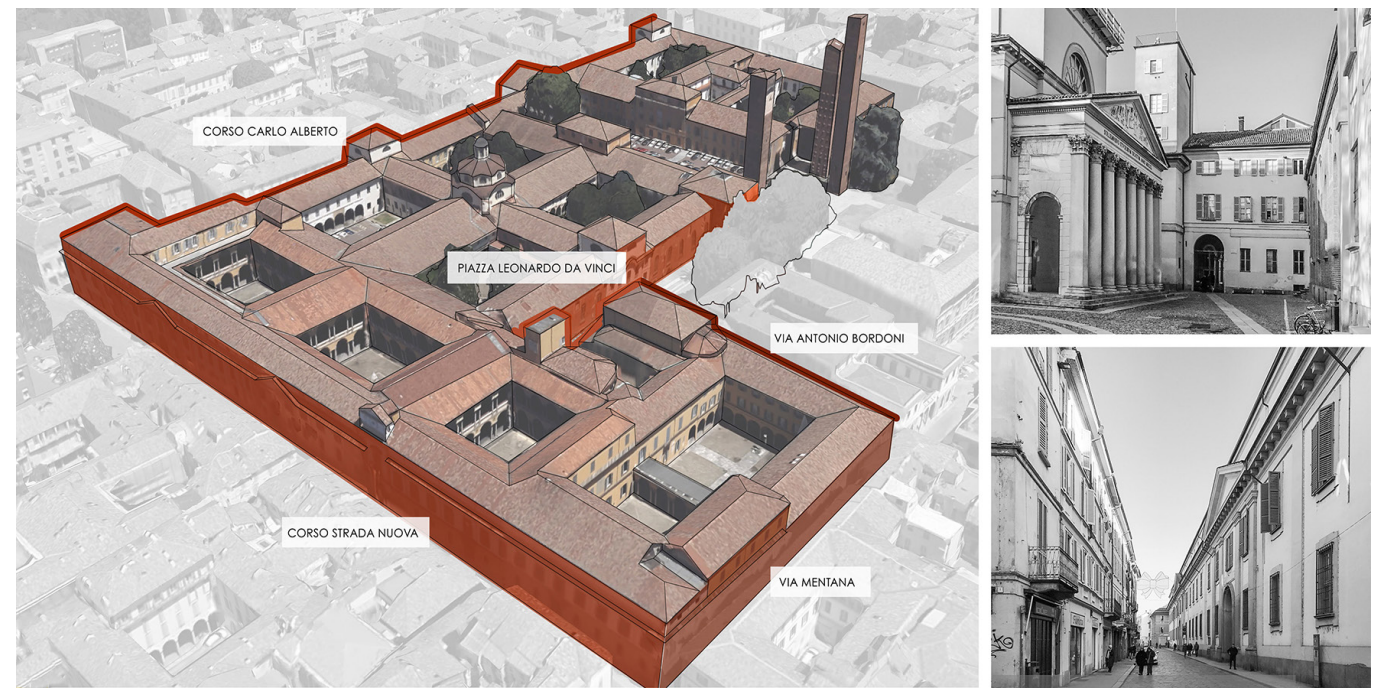

d'Europa, risulta caso emblematico di questo stretto rapporto tra edificio ed ambiente: sin dalla sua fondazione, ha utilizzato spazi appartenenti all'edificato urbano in misura direttamente proporzionale alla crescita della sua importanza e del suo prestigio [8]. In riferimento al Palazzo Centrale, è singolare che tale espansione sia continuata fino agli anni recenti, con l'acquisizione di edifici esistenti, tra loro distaccati, che sono stati riconvertiti ad uso universitario [9]. Questa operazione, che si è interrotta solo alla saturazione del tessuto urbano, testimonia la presenza di un sistema universitario diffuso, che non si limita al complesso del Palazzo Centrale, ma che abbraccia altre strutture sparse nel centro storico, ribadendo con forza il ruolo di primato dell'Università nella città lombarda. Osservando lo sviluppo planimetrico del Palazzo Centrale, è evidente il legame profondo e mimetico con il tessuto storico e, ancora oggi, chiaramente leggibile. Gli ambienti dell'Università vengono vissuti anche al di fuori dell'ambito accademico, sia in quanto spazi di distribuzione delle attività didattiche e culturali, sia nel loro rapporto con la città, definendo un sistema paragonabile a quello dell'isolato urbano o del quartiere, composto di passaggi, carrabili e pedonali, oltre che ad aree aperte per la sosta [ I0]. II Palazzo Centrale rappresenta ancora oggi uno dei principali monumenti di Pavia, emblema della sua identità storica, indissolubilmente legata alla città dal punto di vista storiografico, urbano, sociale ed economico. Legame anche ribadito, in anni recenti, dall'amministrazione di Pavia che "non rinuncia alla possibilità di diffondere la presenza dell'Università, di mescolare studenti e cittadini, di 'sfruttare' il prestigio dell'Università pavese per riqualificare ed arricchire l'intera città" (Documento di indirizzi urbanistici per la redazione del Piano Regolatore Generale di Pavia), [ I l]. Per comprendere appieno questo
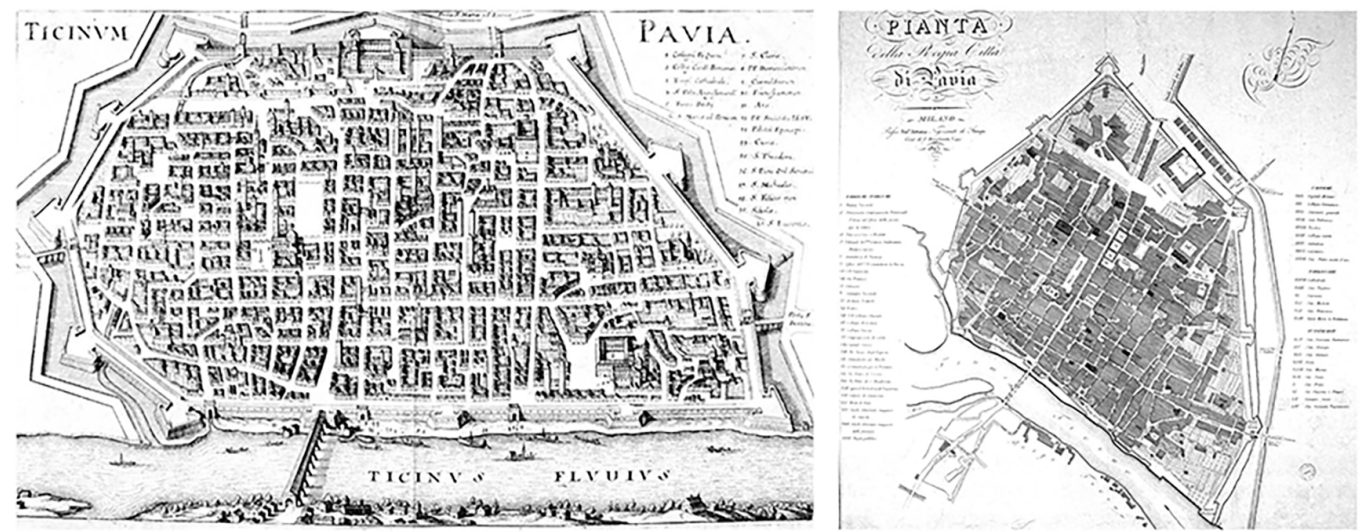
rapporto, la storia della sua edificazione rappresenta un elemento cruciale attraverso alcune delle principali operazioni di trasformazione ed ampliamento che hanno interessato il nucleo storico e l'edificio della Centrale. Osservando lo sviluppo planimetrico risulta evidente la sua naturale partizione in due porzioni, differenti per originaria destinazione e periodo di realizzazione: la prima è caratterizzata dalle numerose edificazioni che si sono susseguite in maniera graduale fino all'800' e si sviluppa seguendo l'andamento di Corso Strada Nuova; la seconda porzione è invece rappresentata dall'annessione, avvenuta nel I95।, dell'antico Ospedale San Matteo [12]

ig. 4. Planimetria della Central punti del Palazzo Centrale dell'Università d Pavia della nuvola di punti. complesso si configura quaprio quartiere incastonato ne essuto urbano.

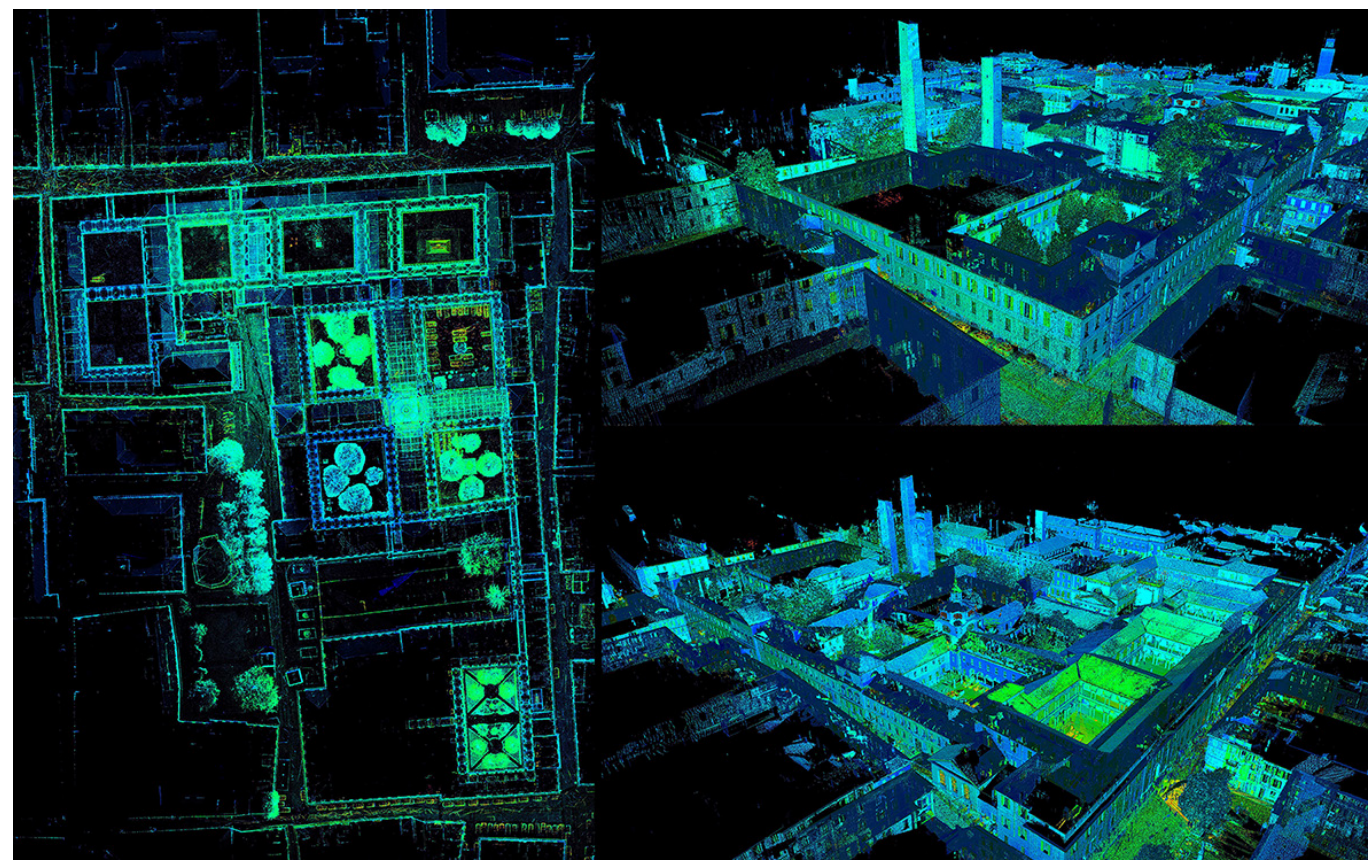

\section{Sviluppo edilizio e urbanistico del Palazzo Centrale}

Lo Studiorum, poi Università di Pavia, venne fondato ufficialmente nel |36| per volere di Carlo V, ma la sua attuale localizzazione nel Palazzo Centrale risale a quasi un secolo dopo [I3]. Nel periodo compreso tra il I 485 e il I 490, fu Ludovico il Moro a concedere l'uso di un palazzo di AzzoneVisconti, prossimo all'Ospedale San Matteo, allora in costruzione, nel cuore della città. II palazzo, che si sviluppava intorno a due cortili (oggi Cortile dei Caduti e Cortile Volta), venne modificato, intorno al I 534, con la realizzazione di portici e loggiati, per il sostegno dei solai lignei esistenti. Durante il regno di Maria Teresa d'Asburgo, I'Università di Pavia vide un periodo di crescita ed espansione che portò, tra il I77I e gli anni '20 dell'Ottocento, ad importanti interventi di modifica ed ampliamento, come la sostituzione dei solai lignei con sistemi voltati a crociera, la realizzazione di importanti ambienti, come l'allora Aula Magna (attualmente Aula Foscolo) e l'espansione verso sud, con la costruzione del Cortile delle Statue e l'annessione degli ambienti dell'ex monastero del Leano, all'epoca già soppresso.

Questi ultimi due cortili erano separati dagli altri ambienti dalla ex Strada delle Catene, elemento che venne incorporato nel complesso solo negli anni '20 dell'Ottocento.

Quest'ultima mantiene ancora oggi una dimensione urbana, essendo un passaggio pedonale fondamentale per il quadrante nord est del centro storico. Strada delle Catene [14] rappresenta oggi la principale via di attraversamento dell'Università, tramite il cortile delle Statue. Essendo percepita come una via urbana, ha mantenuto un ruolo differente da quello degli altri cortili, nonostante tutt'oggi non sia più visivamente riconoscibile la sua funzione originaria. L'ultimo ampliamento realizzato, che ha condotto l'Università alla conformazione attuale, risale al 195 I ed è consistito nell'inglobamento dei cortili e gli ambienti monumentali 

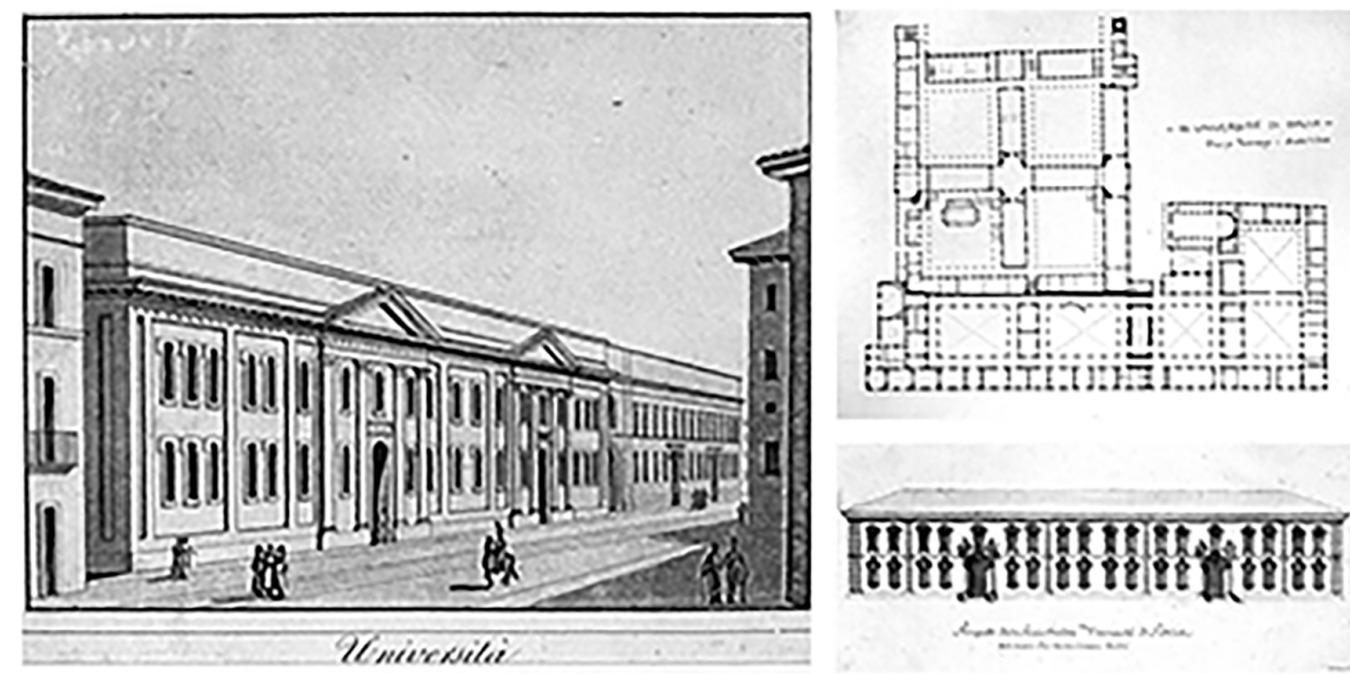

Fig. 5. Lordinamento delle facciate con lo sviluppo di un modulo decorativo che uniforma l'immagine delle numerose fasi di ampliamento lungo

le principali vie della

le prì

rita. I disegni in alto

proposte di intervento

proposte di intervento

sui fronti esterni. In alto
a destra una planimetria

del complesso

risalente al 1945

propone l'imminente

accorpamento degl

ambienti dell'ex

Ospedale San Matteo

(Archivio dei Musei Civici di Pavia).

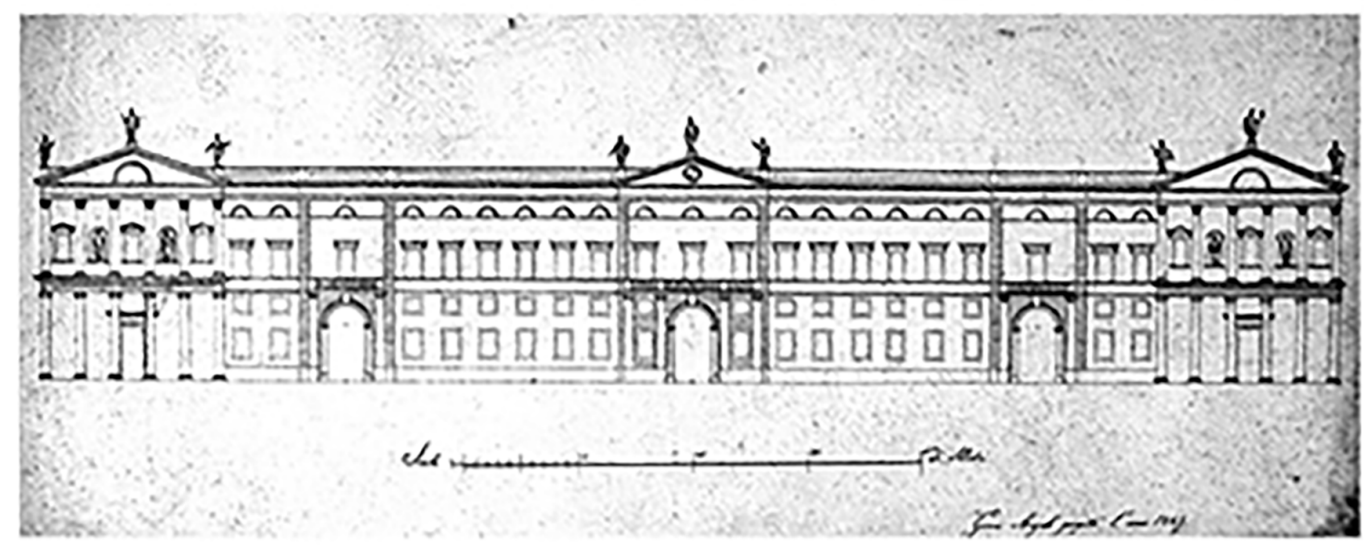

Fig. 6. I fronti strada di Corso Strada Nuova e Corso Carlo Alberto.

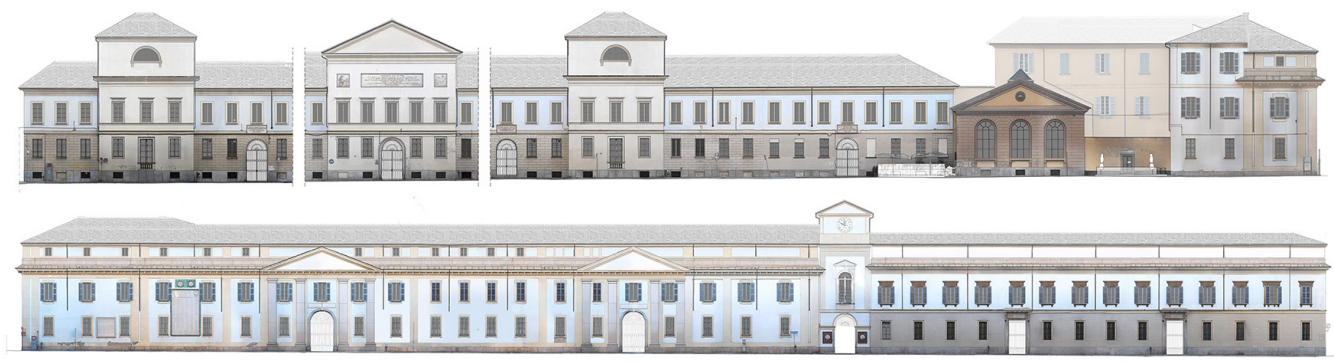


dell'antico Ospedale San Matteo. In seguito a quest'ultima trasformazione, l'edificio della Centrale ha raggiunto la sua conformazione definitiva, e mantenuta fino ad oggi, composta da II cortili che caratterizzano fortemente il suo aspetto e la sua relazione con la città stessa. Emblema del complesso è infatti il continuo susseguirsi di passaggi pedonali utilizzati abitualmente dalla comunità (non solo universitaria) che rende il Palazzo Centrale uno vero e proprio spazio collettivo permeabile e costantemente attivo.

Questa conformazione, caratterizzata dalla giustapposizione di elementi architettonici diversi per epoca e linguaggio, annuncia una ricchezza compositiva, distributiva e di molteplicità di usi che ha come risultato quello di esaltare ancor di più il carattere urbano del Palazzo.

\section{Dal rilievo digitale alla costruzione di banche dati tridimensionali per lo sviluppo di sistemi informativi}

Le attività di rilievo digitale e restituzione grafica dei fronti del Palazzo Centrale dell'Università di Pavia hanno come obiettivo la strutturazione di un database con dati analizzabili su ambiti multidisciplinari e con differenti livelli di approfondimento.

Il processo di documentazione ricopre il ruolo di starter fondante per ogni azione strutturata che si ponga come obiettivo la gestione di ambienti complessi. In questo contesto, la documentazione viene intesa in modo ambivalente sia come la raccolta delle informazioni morfologiche/geometriche sia come un processo per la gestione dei dati e la loro catalogazione in sistemi multi-scala di archiviazione.

La documentazione acquista dunque un duplice valore, comprendendo sia una raccolta delle informazioni che permettono di intervenire sugli aspetti di gestione, che di informazioni per la conservazione, al fine di garantire la salvaguardia del bene architettonico.

Il processo di rilievo e documentazione si è occupato di restituire elaborati vettoriali e materici delle superfici murarie esterne, concentrandosi su ciò che, nelle vie della città e negli

Fig. 7. Immagini dei Cortili interni al Palazzo dell'Università: nella foto da drone sono visibili (dal basso verso l'alto) Cortile delle Statue, Cortile di Volta, Cortile dei Caduti.
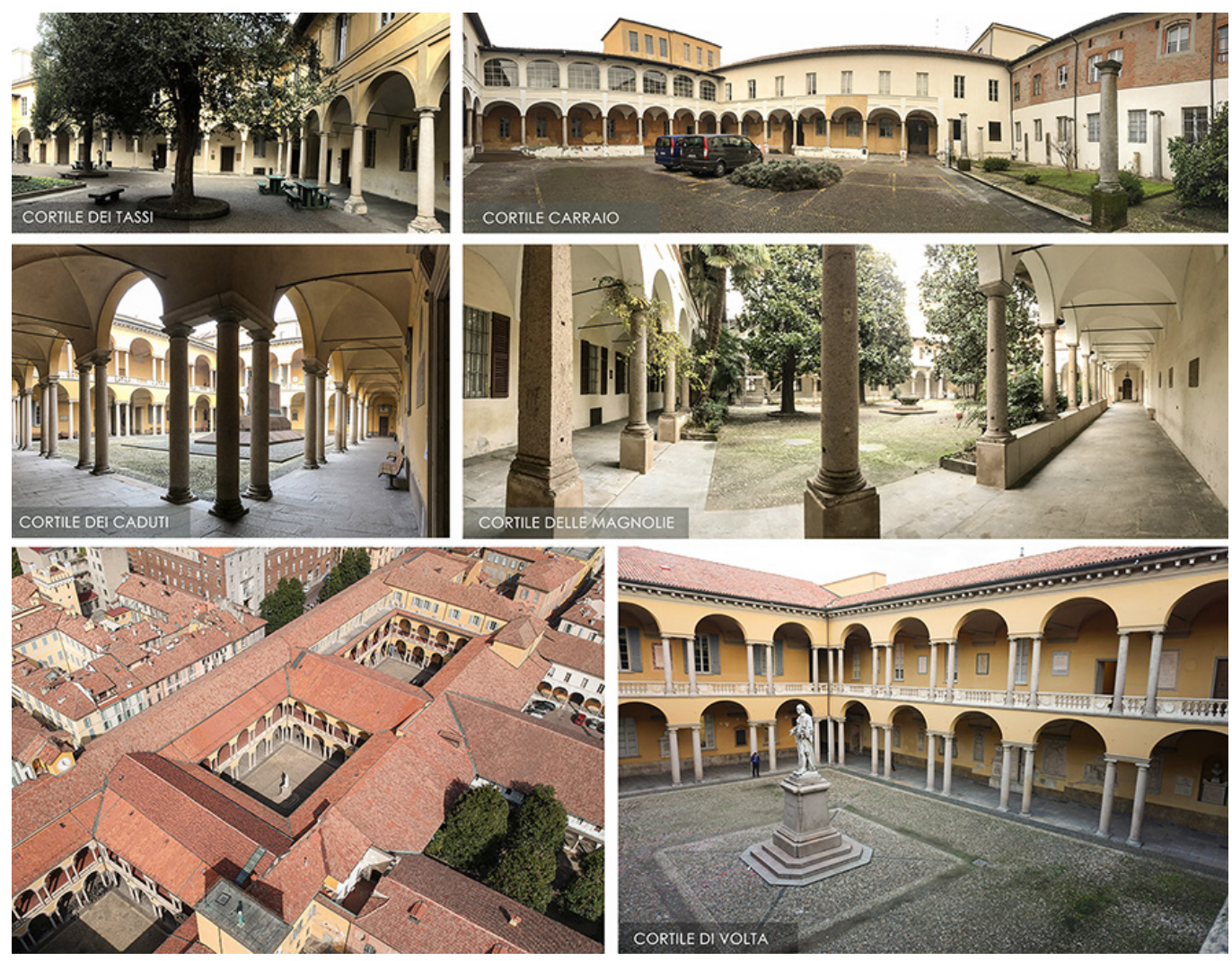
Fig. 8. Sezione ambientale. I cortili sono ambienti interni alla struttura ma attraversabili durante il giorno. A destra il complesso si affaccia su Strada Nuova dove con i suoi 192 metri di lunghezza, si erge di lunghezza, si erge a principale della città
Fig. 9. Vedute della nuvola di punti e sezione passante in corrispondenza della ex Strada delle Catene. L'Università costituisce con le sue permeabilità uno dei nodi cruciali della Città attraverso un passaggio che collega Corso Strada Nuova con Piazza Leonardo da Vinci.

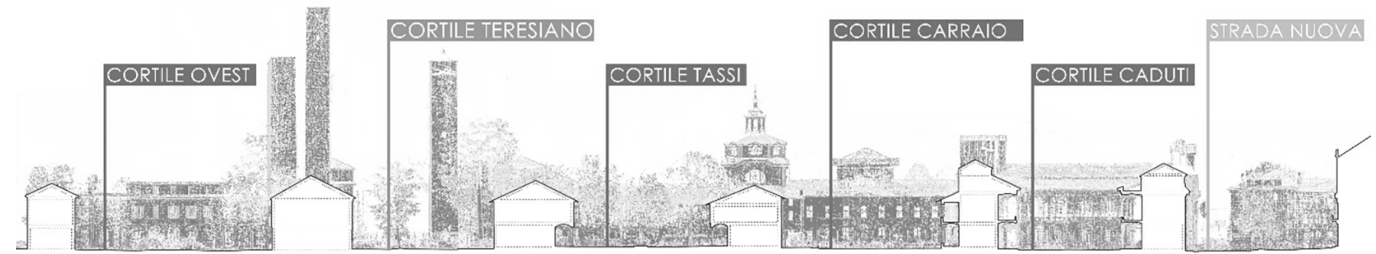

ambienti interni ai cortili, rappresenta la quinta scenica dell'ambiente urbano. L'estensione del Palazzo, con quasi un chilometro di fronti distribuiti lungo il perimetro esterno e con I I cortili su più livelli e attraversati da numerosi passaggi coperti, determina un sistema che, a livello di progettazione delle azioni di rilievo, si connota più a scala urbana che architettonica, senza perdere però l'alto livello di dettaglio necessario a restituire gli elementi tecnologici e gli apparati decorativi.

A partire dai disegni ottenuti sono stati realizzati modelli tridimensionali, volti sia a costituire ambientazioni virtuali che sistemi di gestione parametrica del dato. La duplice finalità dei modelli permetterà di strutturare piattaforme interattive dalle quali strutturare sistemi per produrre simulazioni all'interno dello spazio, interazioni con l'ambiente virtuale ma anche strumenti per programmarne aspetti gestionali. Tra le attività di implementazione di questi modelli e lo sviluppo del progetto di ricerca, vi è la possibilità di integrare i dati raccolti e trasposti sotto forma di schede informative ed elaborati vettoriali con i modelli semantizzati ed inseriti all'interno della piattaforma GIS. Un sistema informativo esteso sull'intero complesso è ciò che permetterebbe agli organi competenti di gestire in maniera strutturata le opere di manutenzione, programmando il loro susseguirsi in base a delle priorità di intervento, e aggiornarle nella piattaforma digitale. La piattaforma informativa, attraverso la quale è possibile gestire facilmente il passaggio di scala da quella urbana a quella architettonica, è integrabile anche con altre metodologie di progettazione dell'edificato, quali BIM o, in caso di beni architettonici, HBIM.
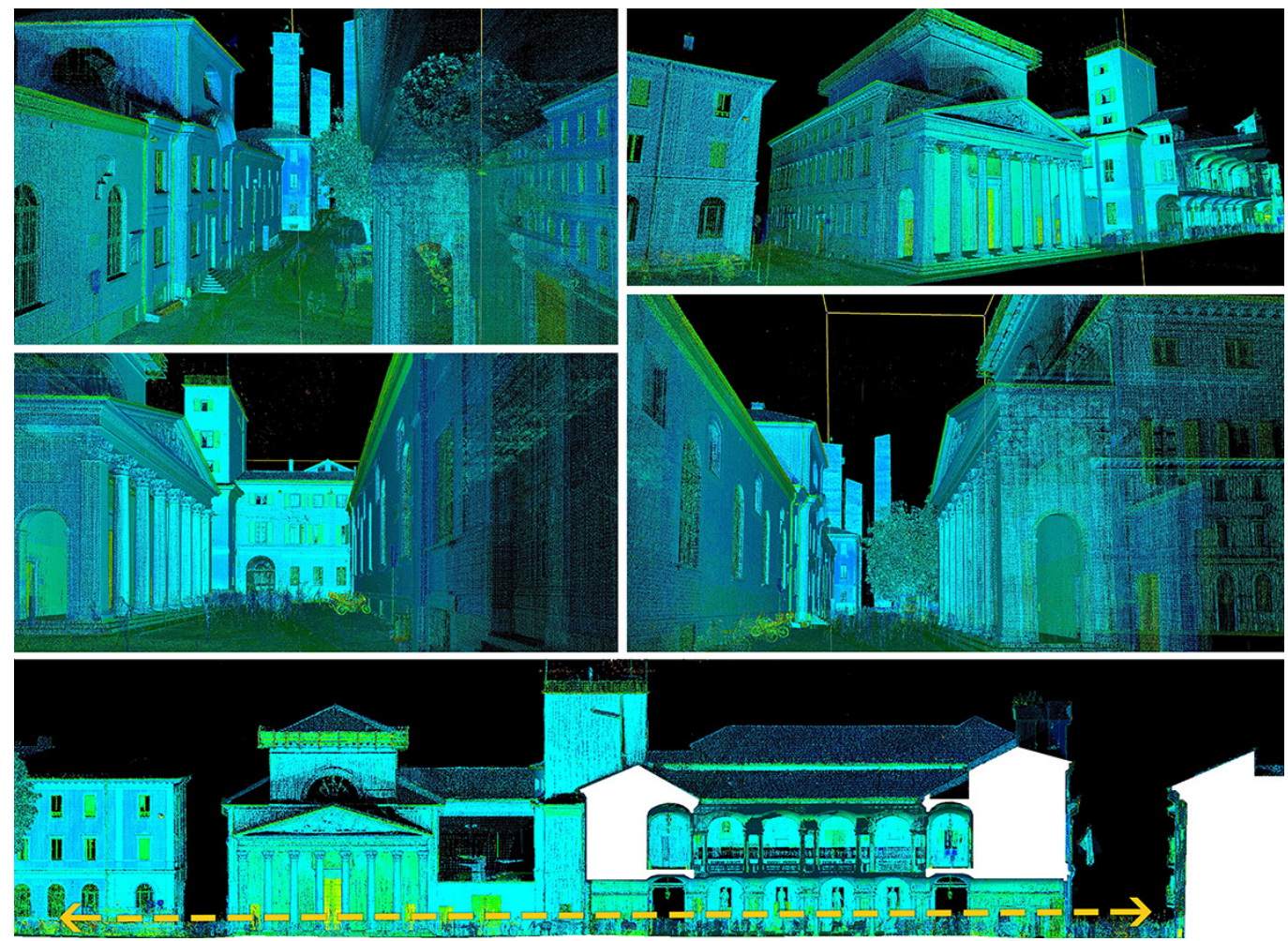
Fig. 10. Fase di

restituzione grafica di una porzione del fronte lungo Corso Strada Nuova: (a sinistra) diseono CAD de fronte urbano: (a destra) gestione della nuvola di

punti per it disegno afilo 'll'
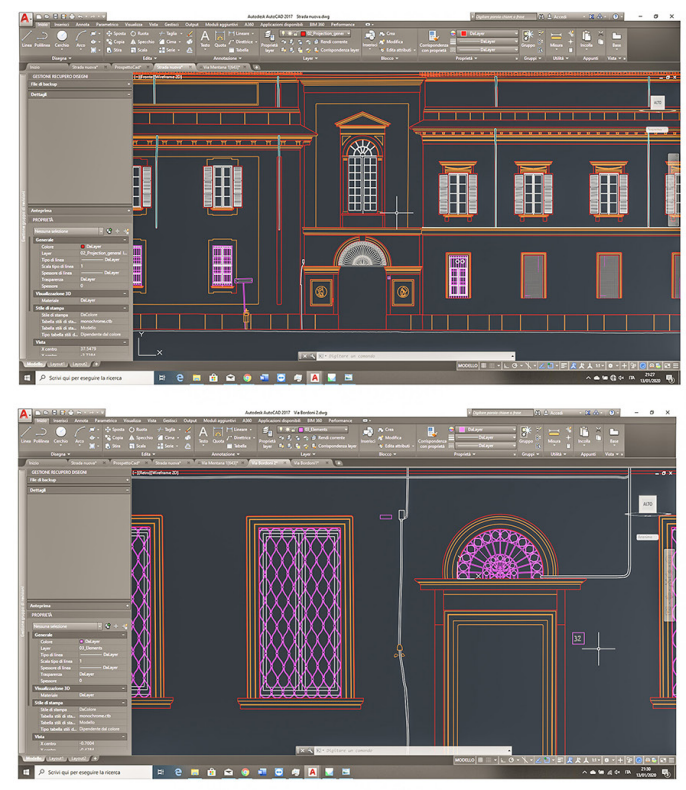
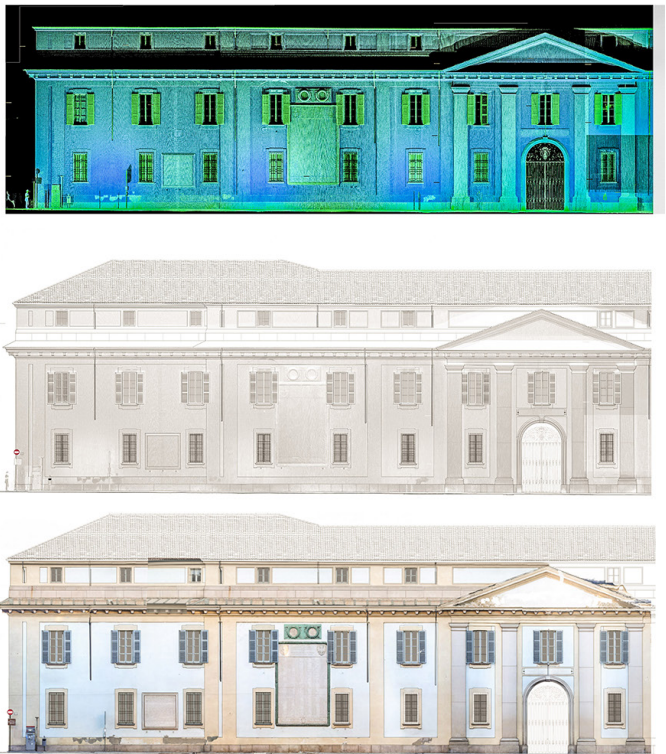

Fig. I I.Viste di modelli di alcuni cortili dell'Università: (a

sinistra) cortile ovest;

(a destra) cortile dei

Tassi. I modelli sono stati

realizzati mantenendo

una scomposizione pe

elementi costruttivi

immaginando uno

sviluppo di piattaforme

interattive e modeli

collaborativi.

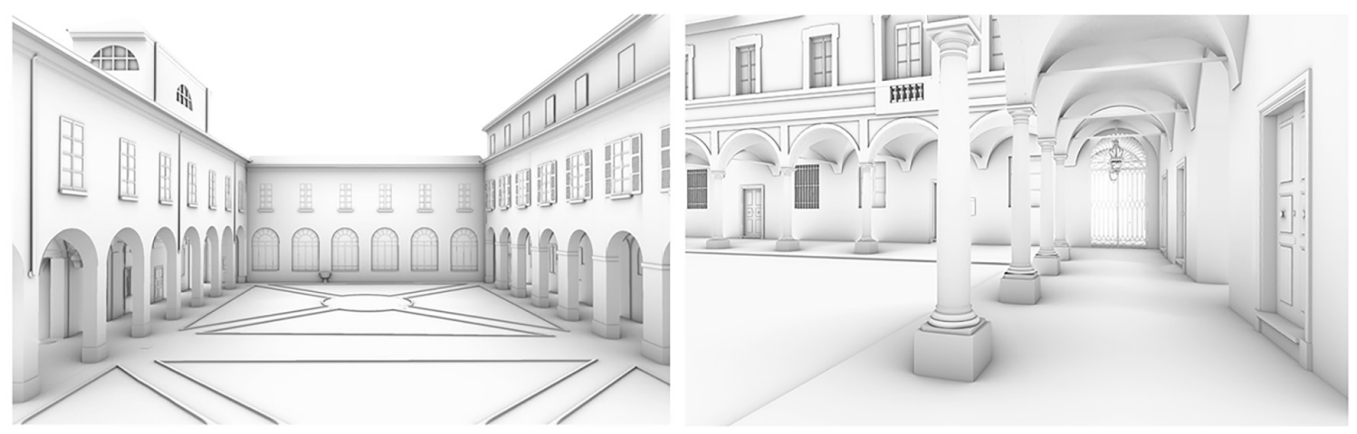




\section{Conclusioni}

Un sistema informativo così strutturato si configura come la cartella clinica del manufatto, e come per un organismo vivente vi è la necessità di aggiornarne costantemente il sistema di lettura e le modalità con cui le informazioni vengono trasmesse in modo da descrivere univocamente le necessità manutentive legate all'edificio.

II sistema informativo diventa uno strumento per leggere, modificare e trasmettere la molteplicità di informazioni legate al bene architettonico e al suo contesto, lette ed interpretate come mezzi per raggiungere gli obiettivi di tutela e gestione, nonché di preservazione dei valori storici e culturali, rafforzando i legami tra la città e il patrimonio costruito.

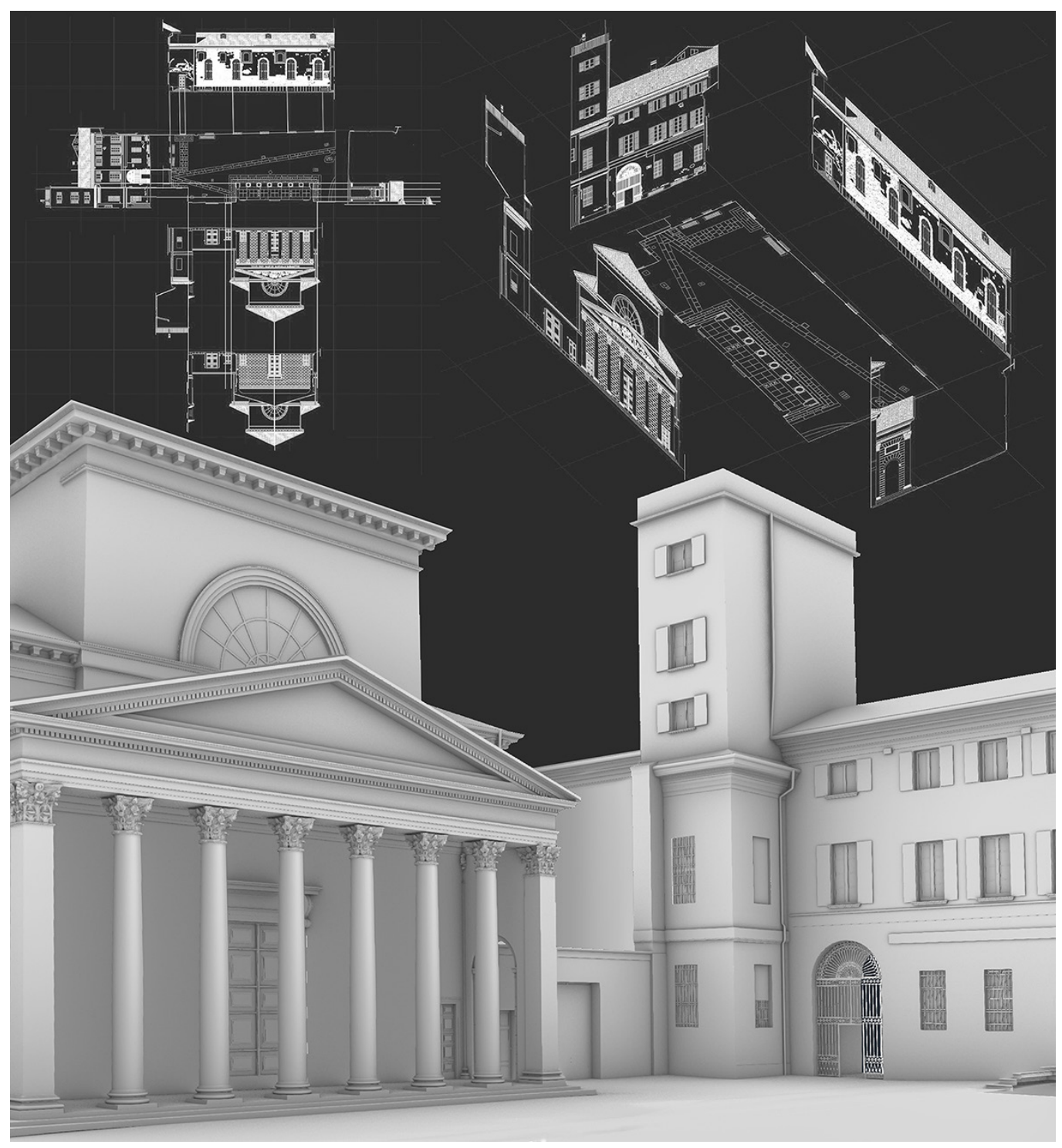




\section{Note}

[I] Si deve la stesura dei capitoli I e 5 a Francesca Picchio, 2 ad Alessia Miceli, 3 e 4 a Elisabetta Doria.

[2] Nel 2006 la Convenzione per la salvaguardia del patrimonio culturale immateriale dell'UNESCO riconobbe come patrimonio da tutelare, oltre al bene stesso intenso come "testimonianza materiale avente valore di civiltà", anche "le tradizioni orali intese come veicolo del sapere, le arti, le pratiche sociali, i riti e le feste, l'artigianato tradizionale, la conoscenza e le pratiche concernenti la natura e l'universo"'[Convenzione Franceschini, per la Salvaguardia dei Beni Culturali, 1967 ].

[3] Nonostante la conservazione dell'elemento materiale risulta lo scopo finale nella maggior parte degli interventi su beni architettonici, vi è consapevolezza che tale azione non sia sufficiente per poter definire il bene stesso valorizzato. "La valorizzazione dell'architettura, ovvero la sua effettiva fruizione culturale, richiede infatti (diversamente da quella di altre categorie di beni) che essa possa essere fisicamente fruita dall'utenza; come afferma Amedeo Bellini: "Un bene non è tale se non è fruibile, la pura contemplazione non appartiene all'architettura" [Morandotti et al. 20 I 4].

[4] "II ricordo è in grandissima parte ricostruzione del passato operata con l'aiuto di dati presi dal presente" [Halbwachs 200 I]. La memoria diviene collettiva e non più singola quando è l'insieme dei ricordi di una collettività nel medesimo gruppo sociale. Non per questo deve essere intesa come somma delle singole memorie individuali, bensì come rielaborazione, perché il passato non viene conservato invariato ma ricostruito nel presente in base ai cambiamenti, alle esigenze e agli interessi, che nel corso del tempo si sono modificati e sono diventati espressione della società presente. II ricordo diventa una visione d'oggi del passato che avviene nell'attualità e ha una dipendenza da essa.

[5] Memoria e forma possono connettersi con legami differenti, ma tutte le forme architettoniche sono una memoria, di loro stesse e delle loro opere di generazione. Rimane il fatto che sia la forma l'indicatore di memoria, e non il contrario, senza dover con questo immaginare la forma dell'architettura come un elemento immutabile. Per approfondimenti: D'Urso 20 I 8.

[6] II progetto di documentazione digitale dei fronti e dei cortili e degli ambienti monumentali del Palazzo Centrale dell'Università degli Studi di Pavia è illustrato nel testo in bibliografia: Morandotti, 2019.

[7] II patrimonio architettonico, in particolare quello religioso, è stato fonte di spazi ed ambienti per l'edilizia universtaria nelle prime fasi della sua espansione: era prassi comune, infatti, ospitare le funzioni universitarie in edifici ecclesiastici, o riadattare questi ultimi, in mancanza di sedi proprie [Lucenti 20।8].

[8] II centro storico di Pavia è una sovrapposizione di layer in cui le espansioni e modifiche legate all'Unviersità sono un elemento caratterizzante [Radice 20I4].

[9] A livello pavese, il sistema degli ambienti universitari posti nel centro storico della città ha da sempre sfruttato il riuso degli spazi ecclesiastici sconsacrati disseminati sul territorio. Anche il Palazzo Centrale ha inglobato un ex edificio ecclesiastico durante l'espansione che ha annesso l'attuale cortile del Leano e del Rettorato. L'ex chiesa del monastero del Leano si affacciava direttamente su Corso Strada Nuova, ed è stata oggetto dei lavori di rifacimento avvenuti tra il XVIII e XIX secolo, che hanno reso irriconoscibile il complesso religioso [Lucenti 20।8].

[10] L'Università è soggetta ad orari di chiusura serali, legati ad un aspetto di sicurezza urbana e prevenzione di vandalismi, ma rimane uno spazio fruibile anche nei periodi di festivi di chiusura e sospensioni delle lezioni o delle attività, garantendo alla Città una continuità di percorsi e flussi naturali all'interno del centro storico.

[I I] Comune di Pavia, Assessorato all'Urbanistica, Documento di indirizzi urbanistici per la redazione del Piano Regolatore Generale di Pavia (Milano, aprile 2002), p. 86.

[12] II Palazzo Centrale dell'Università di Pavia, per come si può apprezzare nella sua interezza oggi, è frutto di numerose addizioni e variazioni che a partire dal |36| hanno caratterizzato il suo ampliamento. Può essere separato in due porzioni differenti per originaria destinazione e periodo storico: la prima porzione è caratterizzata dalle numerose edificazioni che si sono susseguite in maniera graduale nel corso di secoli fino all'800, la seconda porzione è invece rappresentata dall'aggiunta della struttura originaria dello storio Ospedale San Matteo, annesso solamente nel I95 I dopo il definitivo sgombero dei locali e la delibera di cessione all'Università. In seguito a questo ultimo ampliamento, la Centrale ha raggiunto la sua conformazione definitiva mantenuta fino ad oggi, composta al suo interno da I I cortili che caratterizzano fortemente il suo aspetto e la sua relazione con la città stessa, configurandosi come un percorso di passaggi pedonali urbani utilizzati da tutta la cittadinanza.

[13] Già nel 825 venne instituita a Pavia la scuola di retorica per mano dell'imperatore Lotario I. L'università come istituzione per come viene riconosciuta oggigiorno nacque con Galeazzo II Visconti nel | 36I, quando ricevette dall'Imperatore Carlo IV di Lussemburgo il decreto per la fondazione dello Studium Generale, costituito da due nuclei distinti composti da quello dei Giuristi con Diritto Canonico e Civile e quello degli Artisti caratterizzato dagli studi di Medicina, Filosofia e Arti Liberali [Mantovani 2012].

[14] Nel 1785 venne realizzato un terzo cortile, progettato dall'architetto Leopoldo Pollach, destinato allo studio teologico. L'attuale Cortile delle Statue era però separato dal resto dell'Università da una strada, chiamata Strada delle Catene, e fu costruito solamente con tre portici lasciando libero il lato rivolto alla strada per una futura unificazione della struttura. II nome della strada deriva dall'abitudine di chiudere questa con catene, durante la prima fase di edificazione dell'Università, per evitare che transitassero cavalli e carri, dato che rispetto alle strade contigue aveva una dimensione minore e non era adatta a sostenere flussi intensi [Pavesi 1955; Giardini 1830].

\section{Riferimenti bibliografici}

Bartolomucci Carla (2009). Sistemi informativi geografici per la conservazione della città storica. In Giannatasio (a cura di). Antiche ferite e nuovi significati. Permanenze e trasformazioni nella città storica. Workshop Internazionale di Restauro Architettonico e Urbano. Roma: Gangemi editore, pp. 87-96.

Bertocci Stefano, Bua Sara, Parrinello Sandro et al. (20I4). Montepulciano 3D: modelli virtuali per l'urbanistica e lo sviluppo dell'ambiente urbano. In Disegnarecon, 7( I 3), V / I-20, pp. I-20. 
Bertocci Stefano, Parrinello Sandro, Pivetta Michelangelo (20 I 8). Un progetto di rinnovamento urbano a Gerusalemme Est. In Paesaggio Urbano, Progetto-Project, 04, pp. 6-27.

Cullen Gordon (1976). Townscape. (Trad. It., Il paesaggio urbano, morfologia e progettazione, 1976). Bologna: Ed. Calderini.

D'Urso Sebastiano (20I8). II riuso delle memorie dei luoghi. In Minutoli (a cura di). ReUSO VI Convegno Internazionale sulla documentazione, conservazione recupero del patrimonio architettonico e sulla tutela paesaggistica. Messina, I I- | 4 ottobre 20 I8. Roma: Gangemi Editore, pp. 377-388

Erba Luisa (1976). Guida storico-artistica dell'Università di Pavia. Pavia: Fusi Editore.

Franco Giovanna (20 I4). Sostenibilità e patrimonio storico: da un caso studio, nuovi orizzonti di ricerca. In Technè 08, 20 I4, pp. 190- 197. Firenze: Firenze University press.

Giardini Elia (1830). Memorie topografiche dei cambiamenti awenuti e delle opere state eseguite nella R. Città di Pavia sul fine del Secolo XVIII, e nel principio del XIX infino all'Anno MDCCCXXX. Pavia: Stamperia Fusi e Comp.

Halbwachs Maurice (200I). La memoria collettiva. Milano: Unicopli.

Lucenti Simone (20 I 8). II recupero di edifici ex ecclesiastici a uso universitario a Pavia.The refurbishment of former ecclesiastical buildings for university use in Pavia. In Colloquiate 2018. Convegno Ar.Tec., Cagliari 12-14 settembre 2018. Monfalcone (Gorizia): EdicomEdizioni, pp. |08-|2|.

Mantovani Dario (20I2). II lungo cammino dei mercanti di sapienza. Le origini dell'Università di Pavia nella storiografia dal XIV al XX secolo. In Mantovani (a cura di). Almum Studium Papiense. Storia dell'Università di Pavia. Dalle origini all'età spagnola. Tomo I. Origini e fondazione dello Studium generale.Volume I. Milano: Cisalpino Istituto Editoriale Universitario, pp. 29-82.

Morandotti Marco, Besana Daniela, Zamperini Emanuele, et al. (2014). La gestione sostenibile del patrimonio immobiliare tra riuso e valorizzazione. In Della Torre (a cura di). La strategia della Conservazione programmata, dalla progettazione delle attività alla valutazione degli impatti. Proceedings if the International Conference Preventive and Planned Conservation, MonzaMantova 5-9 maggio 20 14. Milano: Nardini Editore, pp. 863-874.

Morandotti Marco, Parrinello Sandro, Picchio Francesca et al. (2019). L'Università di Pavia. I cortili e gli ambienti monumentali. Un progetto di documentazione digitale e sviluppo di sistemi di gestione per la manutenzione programmata. In ReUSO, VII Convegno Internazionale sulla documentazione, conservazione recupero del patrimonio architettonico e sulla tutela paesaggistica. Matera 23-26 ottobre 2019. Roma: Gangemi editore, pp. I31-140.

Pavesi Pietro ( 1897 ) La strada delle catene. Pavia: Stabilimento tipografico succ. Bizzoni (Ristampa: Pavia: Stamperia Fusi e Comp. 1955)

Parrinello Sandro (20 I5). Rilevare, disegnare e organizzare la città digitale. In AAVV. Drawing \& City, Disegno \& Città. Cultura Scienza Arte Informazione, $37^{\circ}$ Convegno Internazionale dei Docenti della Rappresentazione. 17-19 sttembre 2015. Roma: Gangemi Editore, 26I-270.

Parrinello Sandro, Picchio Francesca, Bercigli Monica (2016). La "migrazione" della realtà in scenari virtuali: banche dati e sistemi di documentazione per la musealizzazione di ambienti complessi. In Disegnarecon, Vol. 9 n. I 7, pp. I4. I | |4.8.

Parrinello Sandro, Picchio Francesca, Becherini Pietro et al. (2018). The drawn landscape in 3D databases: the management of complexity and representation in the historical city. In 7th Annual International Conference on urban Studies \& Planning. ATINER'S CONFERENCE PAPER SERIES. Athens: Greece by the Athens Institute for Education and Research, pp. I-26.

Parrinello Sandro, Picchio Francesca, De Marco Raffaella et al. (2019). Documenting the cultural heritage routes. The creation of informative models of historical russian churches on upper kama region. In The International Archives of the Photogrammetry Remote Sensing and Spatial Information Sciences, Volume XLII-2/W I 5, 2019. 27th CIPA International Symposium "Documenting the past for a better future", I -5 September 20 I 9, Ávila, Spain: <https://doi.org/ I 0.5 I 94/isprs-archives-XLII-2-W I 5-887-20 I 9>.

Radice Flavia (20I4). Reuse of deconsacrated churches: the case of Pavia. In XXXVII Curset jornades internacionals sobre la intervenció en el patrimoni arquitectònic. "Patrimoni sacre: permanent innovació". Barcelona: AADIPA.

\section{Autori}

Francesca Picchio, Università degli Studi di Pavia, francesca.picchio@unipv.it

Elisabetta Doria, Università degli Studi di Pavia, elisabetta.doria@unipv.it

Alessia Miceli, Università degli Studi di Pavia, alessia.miceli@unipv.it

Per citare questo capitolo: Picchio Francesca, Doria Elisabetta, Miceli Alessia (2020). Definizione di banche dati e procedure per la valorizzazione del Palazzo Centrale dell'Università di Pavia/Definition of databases and procedures for the valorization of Central Palace of University of Pavia. In Arena A., Arena M., Brandolino R.G., Colistra D., Ginex G., Mediati D., Nucifora S., Raffa P. (a cura di). Connettere. Un disegno per annodare e tessere. Atti del $42^{\circ}$ Convegno Internazionale dei Docenti delle Discipline della Rappresentazione/Connecting. Drawing for weaving relationships. Proceedings of the 42th International Conference of Representation Disciplines Teachers. Milano: FrancoAngeli, pp. 2582-2603. 


\title{
Definition of Databases and Procedures for the Valorization of Central Palace of University of Pavia
}

\author{
Francesca Picchio \\ Elisabetta Doria \\ Alessia Miceli
}

Abstract

In the contemporary discourse about the practice of built heritage conservation the awareness of the value of architectural heritage in the collective memory is the foundation to develop a methodology which leads the survey to protect its architectural characters but also its valorisation. This is particularly true when we deal with architectural heritage which has developed a particular symbiotic relationship with its urban setting, as is the case of the Central Palace of the University of Pavia. The building's architectural development has seen the reuse and remodulation of a number of spaces during the centuries; this process has led to a variety of features which one can appreciate when looking both at the scale of the building - because of its varied configuration and decorations - and at its urban scale - the plan opens and merges itself with the city around it, resulting in it being a fundamental part of the historic core. The documentation process of this complex system of interlocked spaces started in 2019, it is developed by the laboratory Dada Lab of the University of Pavia and aims to deliver a corpus documentorum of the courtyards and external facades of the Palace, in order to achieve a multitude of objectives: provide an overview of the appearance and the conservation status of the building, coordinate and plan its maintenance and develop a system able to promote and transmit over time Pavia's peculiar character of university city.

Keywords

digital documentation, restoration, virtual model.
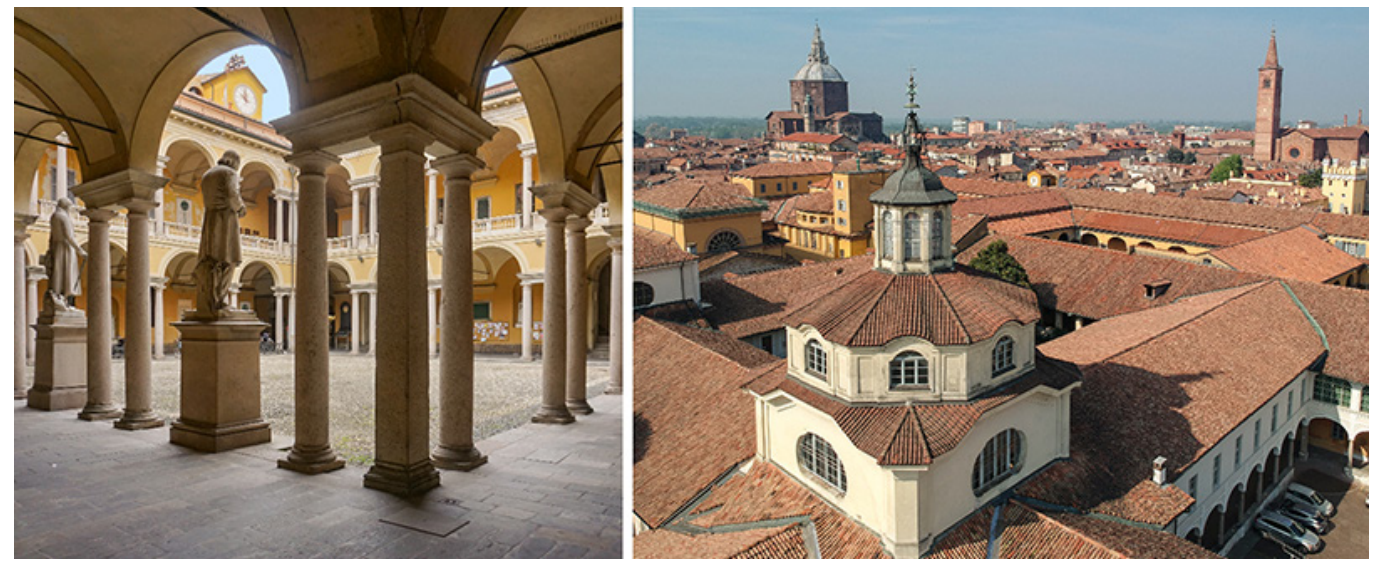


\section{Introduction: memory and documentation for the protection of architectural heritage}

The processes of documentation [I] of architectural heritage is not only its material conservation but also the valorisation of its immaterial [2] value - i.e. its ability of being an element of identity for the city. This awareness is becoming essential in guiding practitioners to interventions able to protect and knowingly manage our built heritage [3]. The community values the built heritage according to its importance in the historical events that involved it and, no less importantly, how they perceive it into a specific setting.

This perception can be influenced by variations in the building's use or transformations in the urban layout that modify the spatial relationship between the building itself and the city. The fruition of urban or architectural space is undermined when severe alterations of the conservation status affect the usability and the ability of the users to cultivate the values related to their identity and its preservation over time [4].

For practitioners dealing with the transformation of the built heritage these considerations instil an awareness that requires, as its starter point, a careful knowledge of the building; in the case of particularly large complexes this analysis sees the building both as an architectural element and as part of the urban system.

This awareness becomes a 'strategy' both as a shared knowledge and a responsibility of actions, guiding of the process of documentation and serving as a tool of preservation of memory [5].

The documentation of the Central Palace of the University of Pavia, fostered by the Technical Department of the University and coordinated by the laboratory Dada Lab of the University of Pavia aims to different objectives: on one side it will deliver, by means of drawing, a graphical representation of all the aspects related to the conservation of its architectural surfaces [6]; on the other side, the project wants to exploit the potential of the newest technology of virtual fruition of space to characterise and populate a digital data base with the all the information needed to achieve a proper knowledge of the building: the process of discretisation and selection of this information will make it possible to amplify its material and immaterial value.

Fig. I. Pictures of the Central Palace of the University of Pavia highlight its relationship with the layout of the historic core.
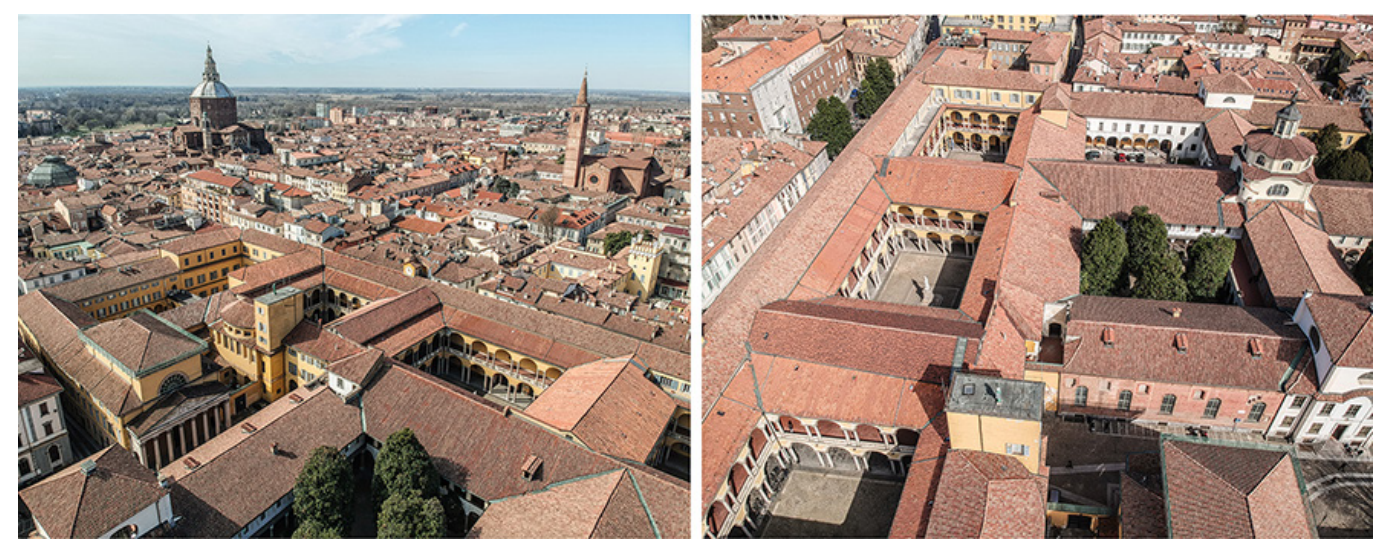

\section{The composite relationship between the City and the University}

One of the most prominent characteristics of Italian universities' architectural assets is their strong bond with their urban layout. One of the reasons at the base of this relationship is the use of different types of built heritage as resources for their expansion, such as private, public, or religious property. In this sense, it is reasonable to affirm that Italian universities were among the first promoters of reuse interventions on the built heritage for new uses [7]. One of Europe's most ancient universities, the University of Pavia is an important 
Fig. 2. The fronts of the monumental complex of the University are urban facades, and recal the composition of their urban setting, with whom they are in a constant Corso Strada Nuova Corso Strada Nuova, outside, encloses severa courtyards which are strictly connected among themselves, but extremely different in their architectural language.
Fig. 3. Historical maps of Pavia show some of the relevant phases of the relevant phases of the In the first oxe (1640) In the first one (1640) the University buildin is concealed into the urban pattern. In the second one (I823) the complex is clearly recognisable thanks to its development around four courtyards; at that time the University had not incorporated the San Matteo Hospital yet (Pavia Civic Museum Archive)
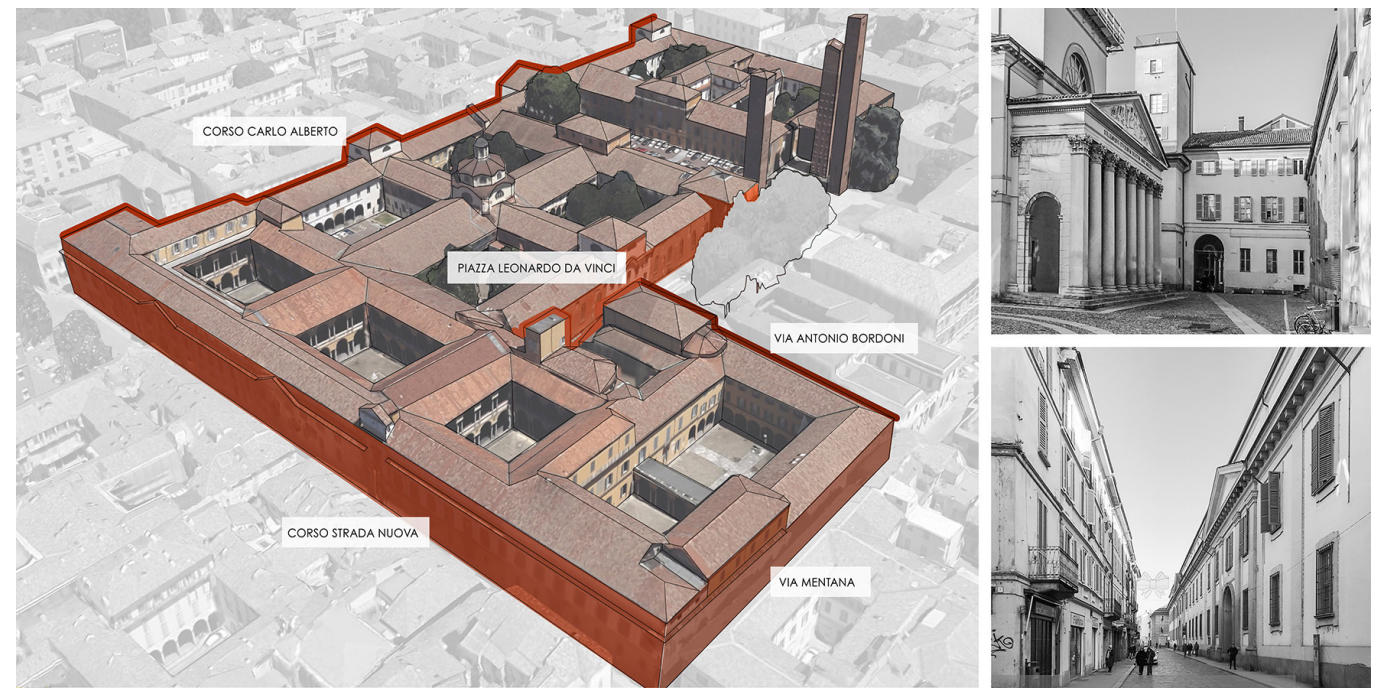

example of this profound bond between buildings and their environment: since the time of its foundation it has been acquiring a number of spaces and buildings, in proportion to its growing importance and prestige [8]. When referring to Central Palace, it is peculiar that this expansion continued until recent years, with the conversion of existent buildings, remodelled and converted in spaces for university-related uses [9]. This process only ended once the urban fabric was saturated and it shows the creation of a diffuse university system, not limited to Central Palace, but comprising a number of properties spread across the historic core, highlighting the primary role of the university in the city.

When looking at Central Palace's plan layout its profound and mimetic bond with the urban fabric is evident and still, clearly legible.The use of the outdoor spaces of the University goes beyond their primal function: they are distribution spaces for the teaching and cultural activities but, most of all, they have an important role in the city, thus defining a system similar to that of urban blocks or neighbourhoods, made of pedestrian and carriageable ways and outdoor areas for the rest [10]. Central Palace is currently one of the main monuments of Pavia, symbol of its historical identity and its relationship with the city encompasses many fields: historical, urban, social and economical. This bond has been recently remarked by the municipality of Pavia which "does not give up the chance to spread the presence of the University, mix students and citizens and 'exploit' the prestige of the University of Pavia in order to improve and enrich the whole city" [Planning guidelines document for the prepa-
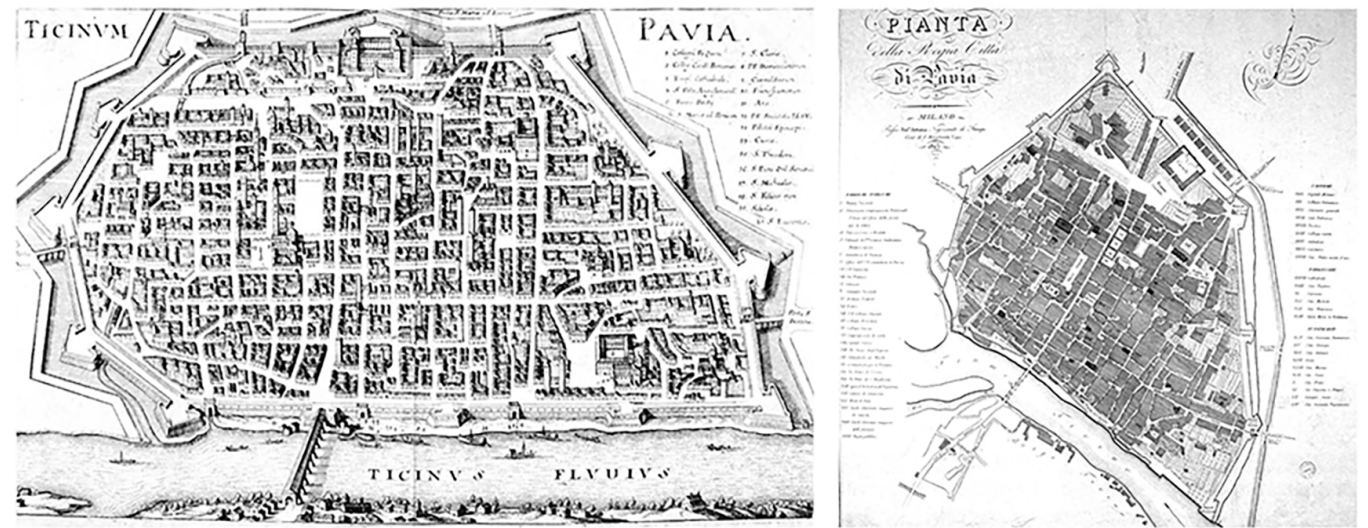
ration of the Pavia General Town Plan] [I I]. The history and the construction phases are fundamental to fully understand this connection since the transformation and expansion processes involve both Central Palace and the historic core. The plan view makes evident the natural partition in two different parts which are different for original use and building period: the first one is made of the several edifications that took place during the years until the 18th century and develops along Corso Strada Nuova; the second one consists of the San Matteo building, which had been annexed to the complex in |95। [12].

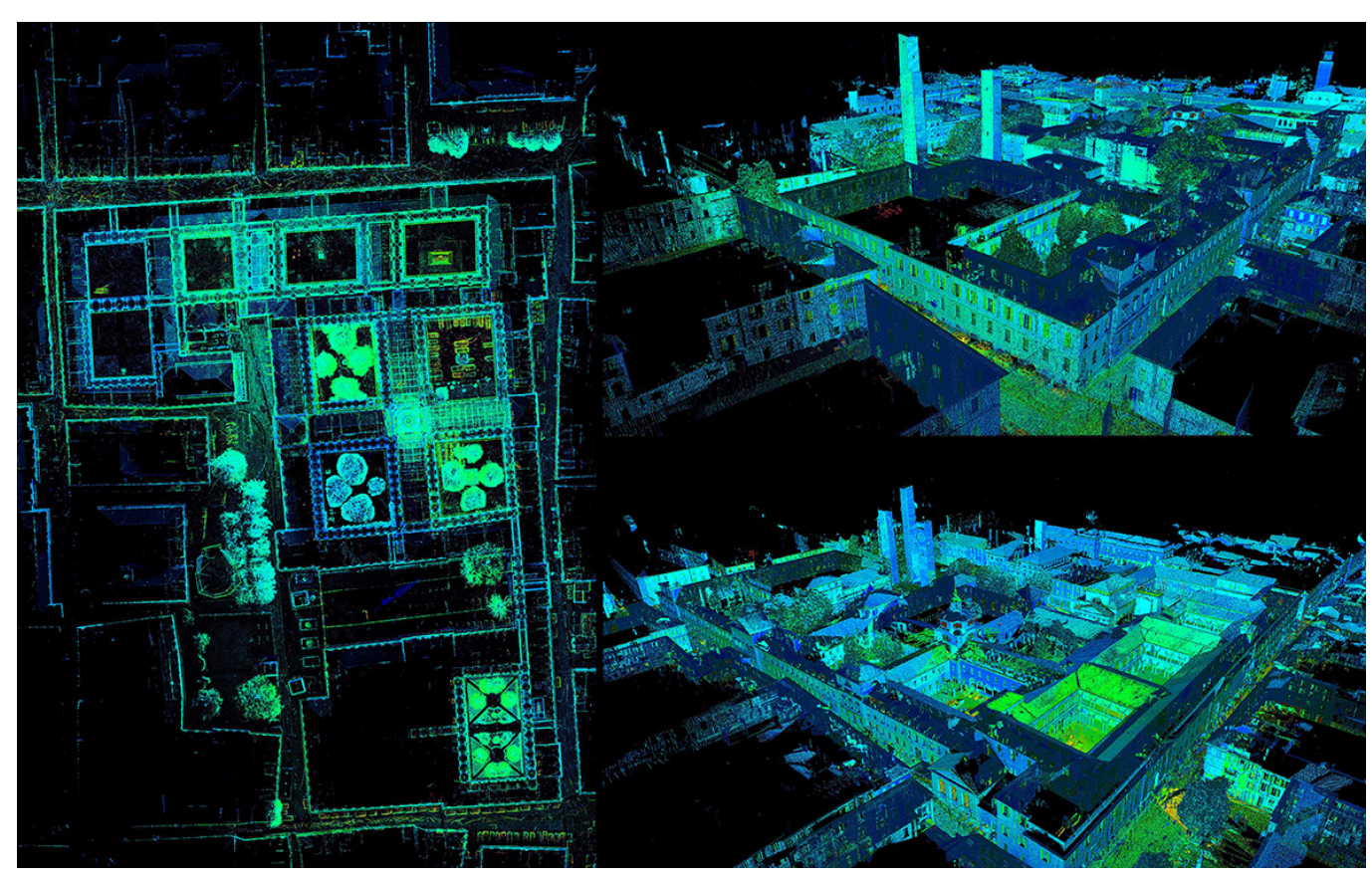

\section{Building and urban development of the Central Palace of the University}

The Studiorum, today the University of Pavia, was officially founded in I $36 \mid$ at the behest of Charles V, but its current location in the Central Palace dates back almost a century after that date [I3]. Between I485 and I490, Ludwig the Moor granted the use of a palace of Azzone Visconti, next to the San Matteo Hospital, at the time under construction, in the heart of the city. The palace developed around two courtyards - now Cortile dei Caduti (Courtyard of the Fallen) and Cortile Volta (Courtyard of Volta) - and was modified, around I534, with the construction of porches and loggias, to support the existing wooden floors. During the reign of Maria Theresa of Habsburg, the University of Pavia experienced a period of growth and expansion that led, between $177 \mathrm{I}$ and the 1920s, to important changes and enlargements.

The variations were the replacement of the wooden floors with systems turned on a cruise, the construction of important spaces, such as Aula Magna (currently the Foscolo Hall) and the expansion to the south, with the construction of Cortile delle Statue (Courtyard of the Statues) and the annexation of the rooms of the former monastery of Leano, which had already been abolished at the time.

These two courtyards were separated from the others by the former Strada delle Catene (Chain Road), an element that was incorporated into the complex only in the I820s.

This road still maintains an urban dimension, being a key pedestrian crossing for the northeast quadrant of the old town. The road [14] is now the main crossing route of the Univer- 

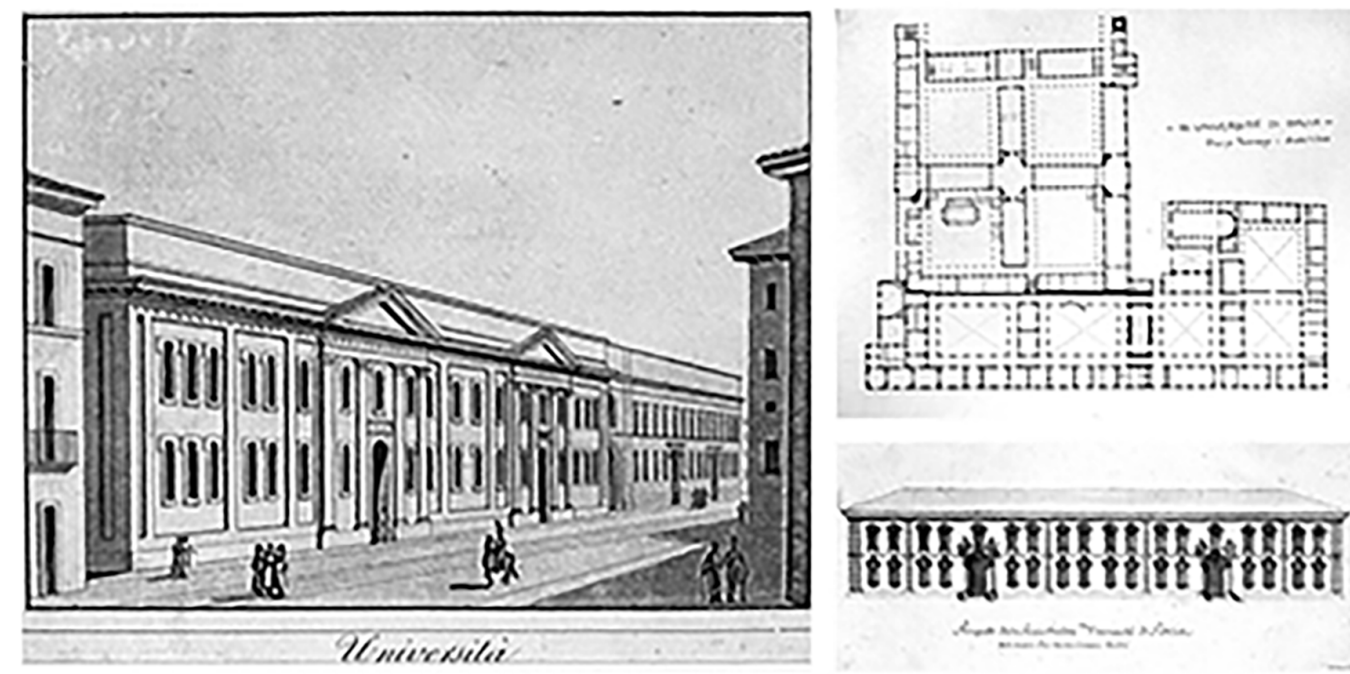

g. 5. Over the years developed some design solutions to standardize the fronts of the Centra Palace of the Univensity Palace of the University that were expanding of the city. The technical drawings represent some proposals for intervention on the external fronts. On the top right, a floor plan of the complex dating back to 1945 proposes the imminent incorporation of the rooms of the former San Matteo

Hospital. (Pavia Givic

Museum Archive).

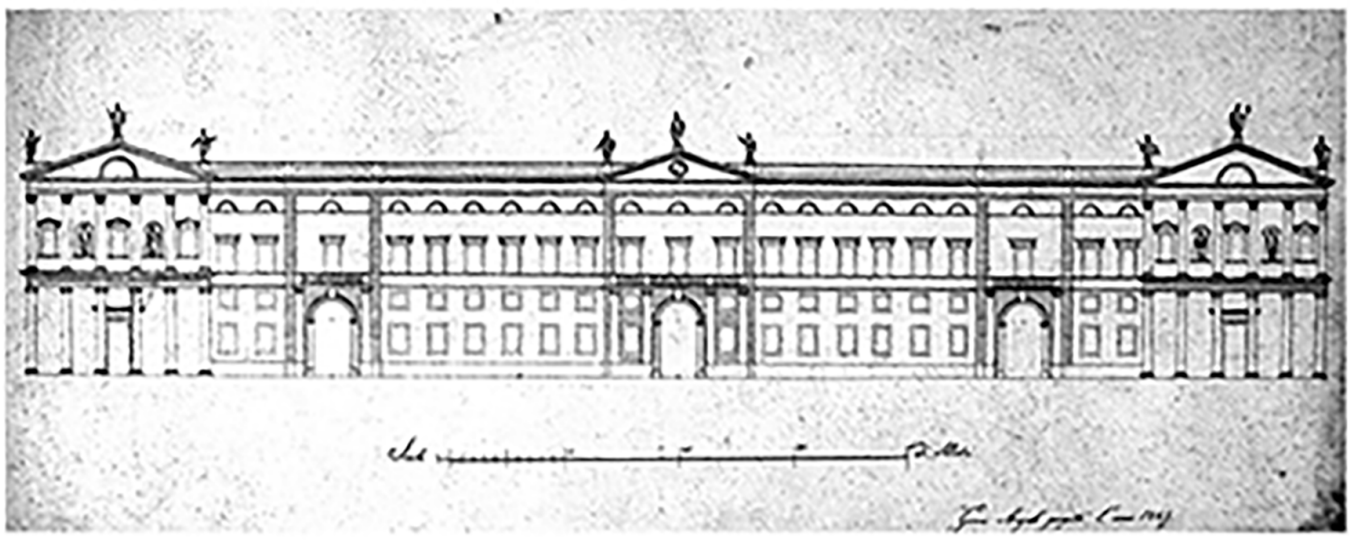

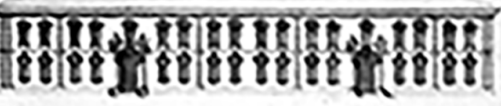

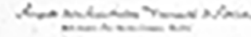

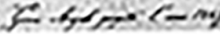
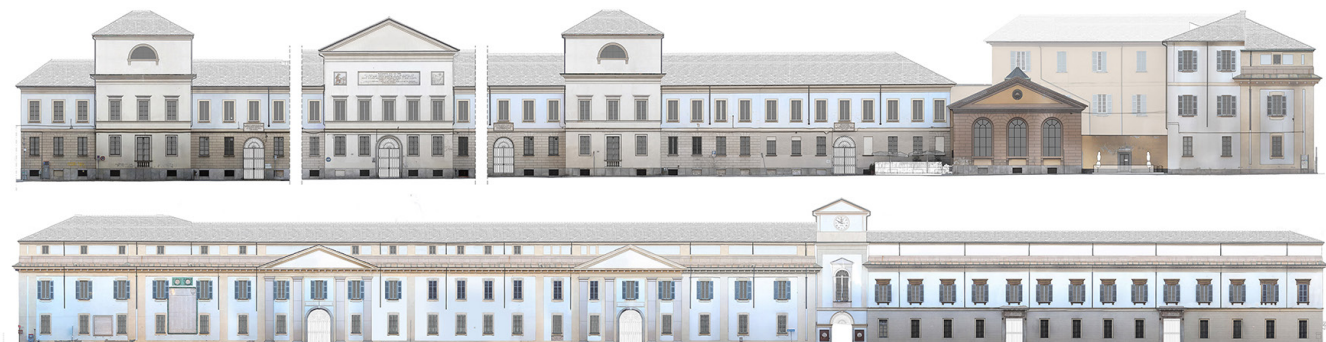
sity, through the courtyard of the Statues. The road is perceived as an urban street and has maintained a different role compared to the other courtyards, although today its original function is no longer visually recognizable.

The last extension was the integration of the courtyards and the monumental spaces of the ancient Hospital San Matteo. This led the University to its current conformation, dating back to 1951.

Following this latest transformation, the Central building has reached its final conformation. To this day it is made up of I I courtyards that strongly characterize its appearance and its relationship with the city itself. Emblem of the complex is the continuous succession of pedestrian crossings usually used by the community (not only in the university area) that makes the Central Palace a real permeable and constantly active collective space.

This conformation, characterized by the juxtaposition of different architectural elements for epoch and language, announces a richness of composition, distribution and multiplicity of uses that results in exalting once again the urban character of the Palace.

\section{Conclusion}

The activities of digital survey and graphic drawings of the fronts of the Central Palace of the University of Pavia aim to structure a data base with data that can be analysed on multidisciplinary fields and with different levels of deepening.

The documentation process is the foundational starter for any structured action that aims to manage complex environments. In this context, the documentation is ambivalently understood both as the collection of morphological/geometric information and as a process for managing data and cataloguing it in multi-scale storage systems.
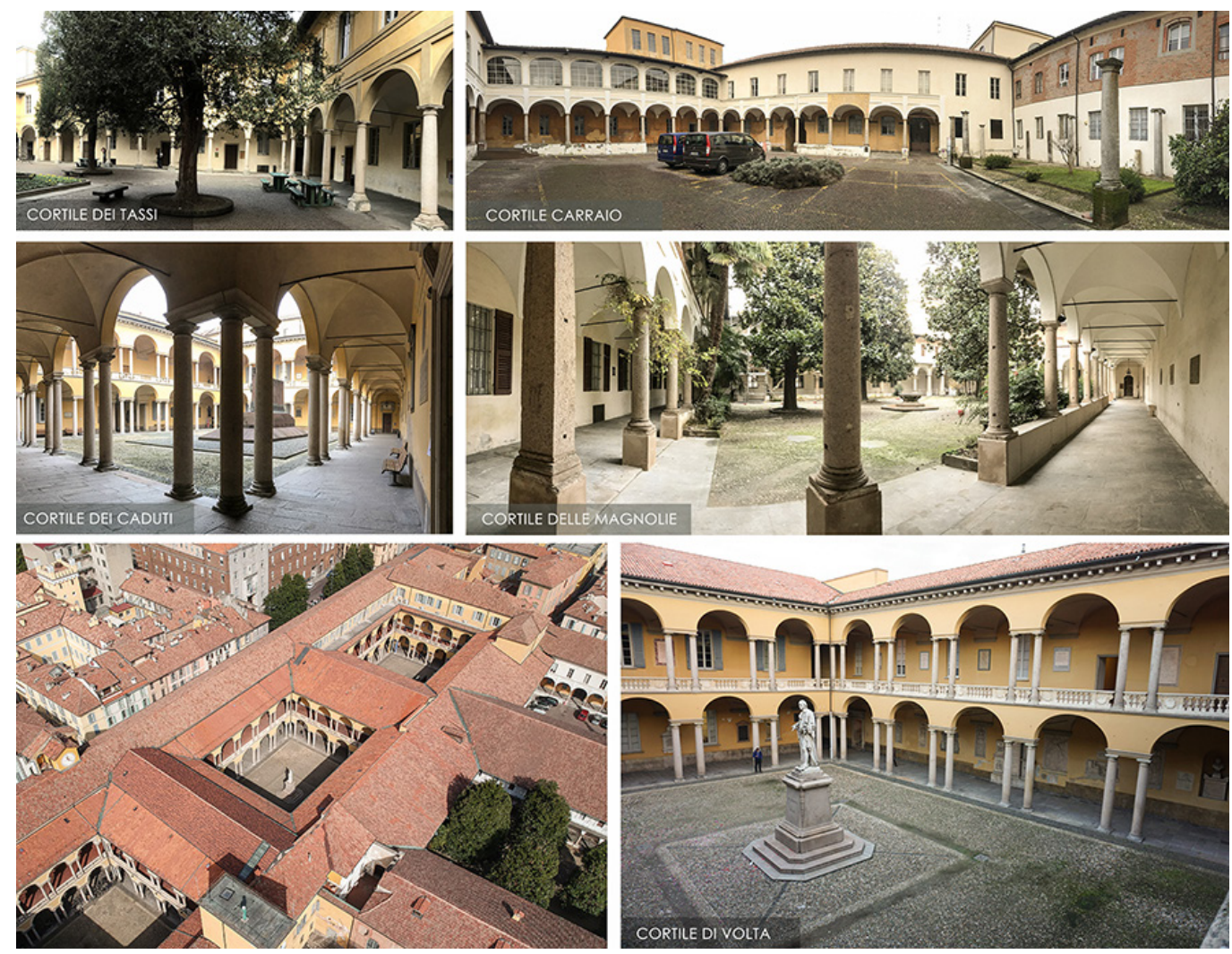
Fig. 8. The landscape section in the figure highlights the layout of the Central Palace complex and how it relates to the city and its urban functions. To the right, the complex overlooks Strada Nuova where, with its 192 meters, the University stands as monument to the city's main street.
Fig. 9. Landscape Section from laser scanner point cloud. The section at the bottom of the image highlights how Strada delle Catene is a natural passageway for users. It connects Corso Strada Nuova with Piazza Leonardo da Vinci, one of the crucial nodes of the City, characterized by the presence of monumenta medieval towers.

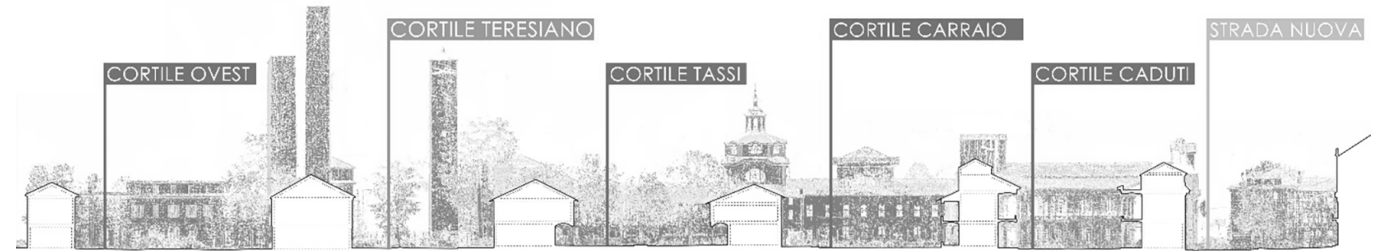

The documentation acquires a dual value, including both a collection of information that allows to intervene on the aspects of management, as well as information for conservation, in order to ensure the preservation of the architectural good.

The survey and documentation process elaborated vector and material works of the external masonry surfaces, focusing on what, in the streets of the city and in the interiors of the courtyards, represents a scenery flat of the urban environment.

The dimension of the Palace, with almost a kilometre of fronts spreading along the perimeter and with I I courtyards on several levels and crossed by numerous covered passages, determines a system that, at the level of the management of the important actions, is connoted to the architectural scale, but without losing the high level of detail needed to return the technological elements and the decorative equipment. From the drawings obtained it has been possibile to make three-dimensional models, aimed to the construction of virtual settings and parametric data management systems. The dual purpose of the models will allow to structure interactive platforms from which produce simulations within space, interactions with the virtual environment but also tools to program management aspects. Among the activities of implementing these models and the development of the research project, there is the possibility to integrate the collected and transpose data in the form
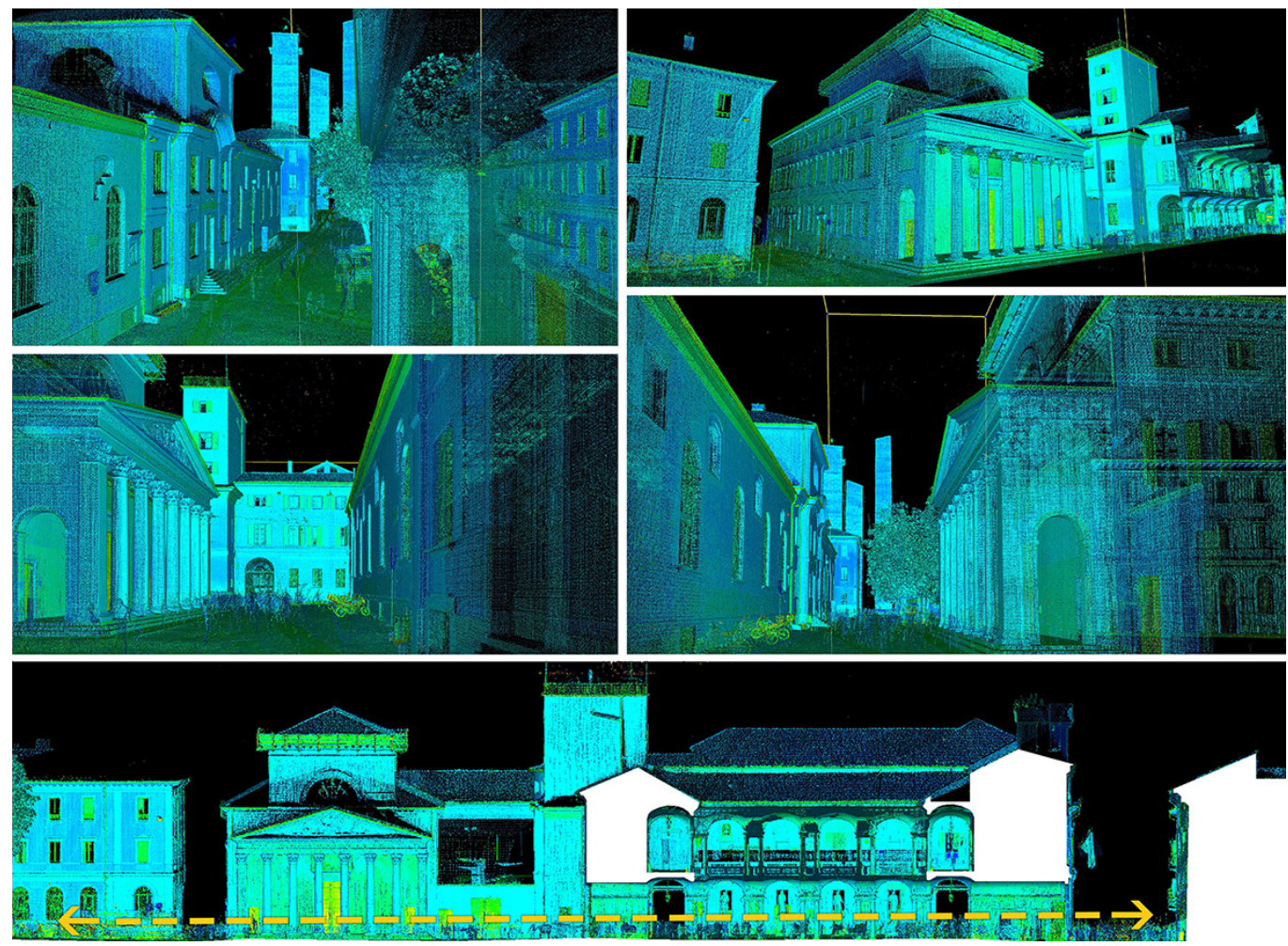
Fig. I0. Representation and drawing phase of a portion of the front alon Corso Strada Nuova:

(on the left) CAD design of the urban front; (on

the right) point cloud

handling for 'iron wire'

design and return of the elaborate material.

Fig. I I.Views of NURBS models of some of the University's courtyards: (on the left) Cortile Ovest (Western

Courtyard): (on the

right) Cortile dei Tassi

Courtyard of the

Cous

modelled according

a simplification

ratio, and maintain the

decomposition in similar

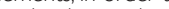

develop interactive

platforms in the future.
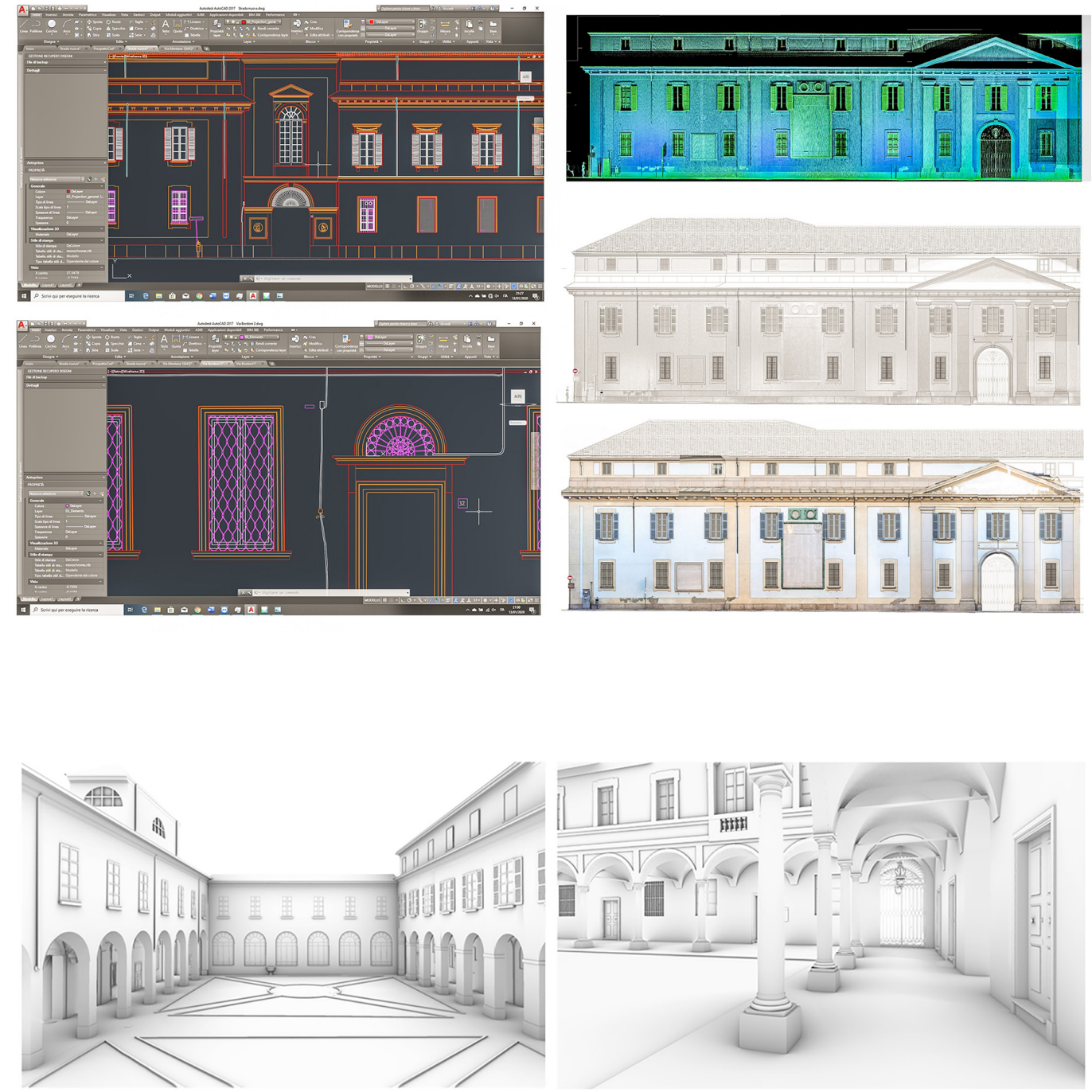
of information sheets and processed vectors with the semantized models and insert them within the GIS platform.

An extensive informative system of the whole complex is what would allow the competent bodies to manage the maintenance works in a structured way, planning their succession according to intervention priorities, and updating them in the digital platform. The informative platform, through which you can easily manage the transition of scale from the urban one to the architectural one, can also be integrated with other methodologies of building design, such as BIM or, in the case of architectural heritage, HBIM.

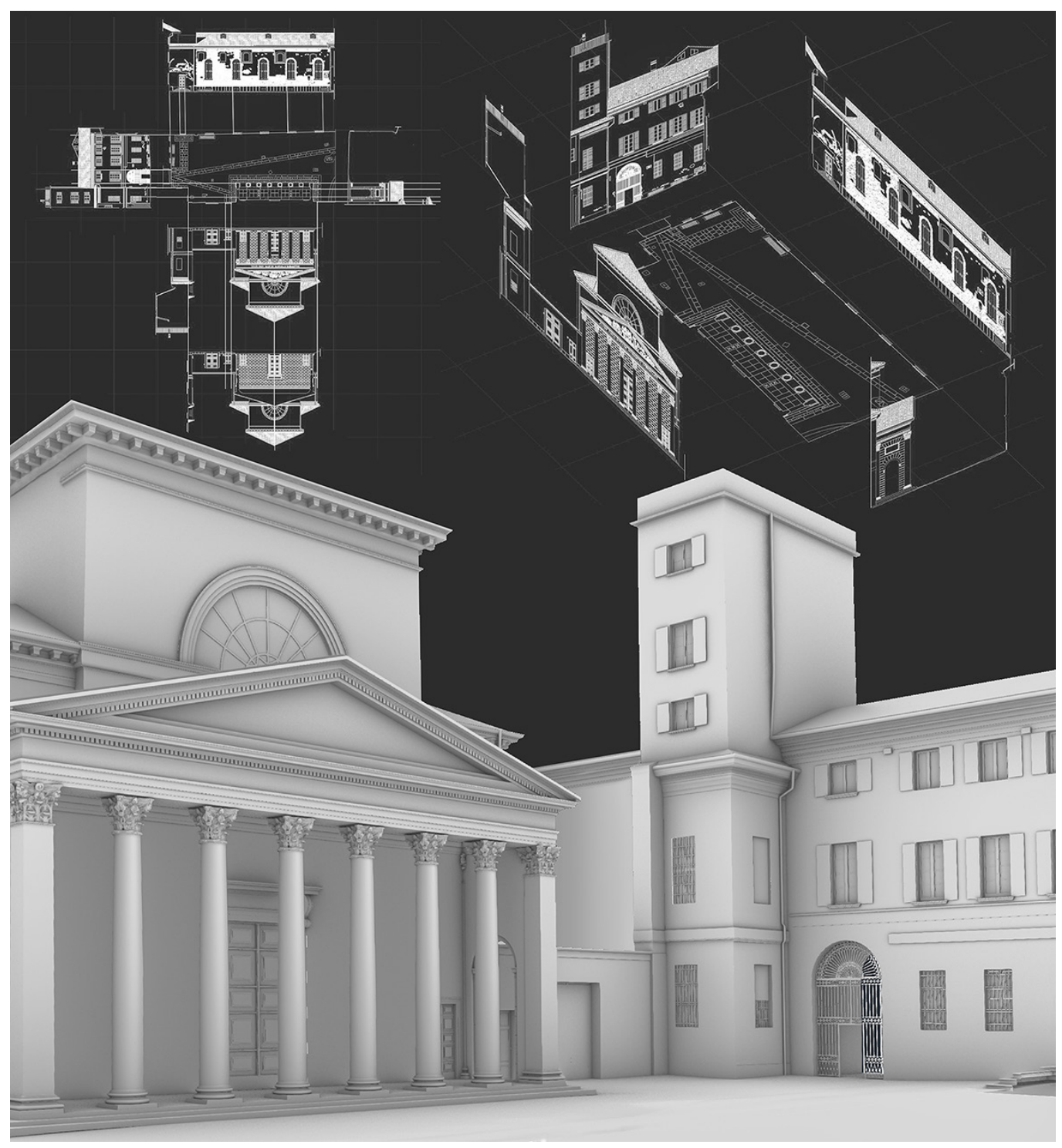




\section{Notes}

[I] Chapter I and 5: Francesca Picchio, Chapter 2: Alessia Miceli. Chapter 3 and 4: Elisabetta Doria

[2] In 2006, UNESCO's Convention for the Safeguarding of the Intangible Cultural Heritage recognized that the material heritage is to be protected, since it represents as a "material witness with the value of civilisation" but also the "living cultural heritage usually expressed in one of the following forms: oral traditions; performing arts; social practices, rituals and festive events" [Franceschini Convention for the safeguarding of Cultural Heritage, 1967].

[3] The conservation of the material aspect is usually the main scope of interventions on the built heritage, but the awareness that this action alone is not sufficient for the valorisation of the heritage itself arises. "The valorisation of architecture, intended as its cultural significance, needs that it must be physically used by the users, differently from other types of heritage: according to Amedeo Bellini: "Heritage is not such unless it is used, the mere contemplation does not belong to architecture". [Morandotti et al. 2014].

[4] "Memory is in great part a reconstruction of the past elaborated from data belonging to the present" [Halbwachs 200 I] Memory becomes collective when it is the collection of memories of a community belonging to the same social group. This does not mean that it is the sum of the single individual memories, but it comes from a re-elaboration. In fact, the past does not persist as invariant but it is rebuilt in the present according to changes, needs and interests that evolved over time and became the expression of the current society. Memory is a vision of the past in today's time and depends on it.

[5] Memory and form can connect to each other with different bonds, but every architectural form is a memory of themselves and their creation process. The form is the indicator of the memory and not the contrary, but this does not mean that the architectural form is immutable. For further info: D'Urso 2018.

[6] The project of digital documentation of the courtyards and the facades of University of Pavia's Central Palace is further explained in the paper in references: Morandotti, 2019.

[7]The built heritage, particularly the religious one, used to be a source for university buildings especially during the first phases of their expansion: it was common use to host university-related activities in religious buildings, or remodel the latter when in lack of proper spaces [Lucenti 20।8].

[8] Pavia's historic core is a stratification of layers with expansions and modifications of the University strongly characterising it [Radice 2014]

[9] The system of the university's buildings in the historic core of Pavia has been reusing ecclesiastical spaces spread across the city since its foundation. The Central Palace incorporated a religious building in the expansion which led to the annexion of Cortile del Leano (Courtyard of Leano) and Cortile del Rettorato (Courtyard of the Chancellor). The ex-church of the Monastery of Leano faced Corso Strada Nuova; today the façade is not recognizable anymore, because of the massive remodelling interventions occurred between the 18th and the 19th century [Lucenti 20।8]

[10]The University outdoor areas are closed during the night, but they are accessible even during holiday and festive periods, thus granting the community with a continuous network of pedestrian passageways through the historic core.

[I I] Comune di Pavia, Assessorato all'Urbanistica, Documento di indirizzi urbanistici per la redazione del Piano Regolatore Generale di Pavia (Milan, April 2002), p. 86.

[12] University of Pavia's Central Palace is the result of a number of additions and modifications that, starting from I36 | characterised its development. After the 1951 enlargement, the Palace reached its final configuration which it had maintained until today: it develops around I I courtyard which strongly affects its appearance and its relationship with the city, and has as a series of passageways widely used by all the citizens.

[13] As early as 825, the School of Rhetoric was established in Pavia at the hands of The Emperor Lotario I. The university as an institution, as it is recognized today, was born with Galeazzo II Visconti in I36 I, when he received from Emperor Charles IV of Luxembourg the decree for the foundation of the General Studies, consisting of two distinct areas of study: the one of Jurists with Canon and Civil Law and the one of the Artists, characterized by the studies of Medicine, Philosophy and Liberal Arts [Mantovani 2012].

[14] In 1785 a third courtyard was built, designed by the architect Leopoldo Pollach, intended for the theological study. The current Cortile delle Statue (Courtyard of the Statues), however, was separated from the rest of the University by a road, called Strada delle Catene (Chain Road), and it had been built only with three porches, leaving the side facing the road for a future unification of the structures. The name of the road comes from the habit of closing it with chains, during the first phase of construction of the University, to prevent horses and wagons from passing, since compared to the adjacent roads, it had a smaller size and was not suitable for intense flows [Pavesi 1955; Giardini 1830].

\section{References}

Bartolomucci Carla (2009). Sistemi informativi geografici per la conservazione della città storica. In Giannatasio (a cura di). Antiche ferite e nuovi significati. Permanenze e trasformazioni nella città storica. Workshop Internazionale di Restauro Architettonico e Urbano. Roma: Gangemi editore, pp. 87-96.

Bertocci Stefano, Bua Sara, Parrinello Sandro et al. (2014). Montepulciano 3D: modelli virtuali per l'urbanistica e lo sviluppo dell'ambiente urbano. In Disegnarecon, 7( I 3),V / I-20, pp. I-20.

Bertocci Stefano, Parrinello Sandro, Pivetta Michelangelo (20 I 8). Un progetto di rinnovamento urbano a Gerusalemme Est. In Paesaggio Urbano, Progetto-Project, 04, pp. 6-27.

Cullen Gordon (1976). Townscape. (Trad. It., Il paesaggio urbano, morfologia e progettazione, 1976). Bologna: Ed. Calderini. 
D'Urso Sebastiano (20/8). II riuso delle memorie dei luoghi. In Minutoli (a cura di). ReUSO VI Convegno Internazionale sulla documentazione, conservazione recupero del patrimonio architettonico e sulla tutela paesaggistica. Messina, I I- I 4 ottobre 20 I 8. Roma: Gangemi Editore, pp. 377-388.

Erba Luisa (1976). Guida storico-artistica dell'Università di Pavia. Pavia: Fusi Editore.

Franco Giovanna (20 I4). Sostenibilità e patrimonio storico: da un caso studio, nuovi orizzonti di ricerca. In Technè 08, 20 I4, pp. 190- 197. Firenze: Firenze University press.

Giardini Elia (1830). Memorie topografiche dei cambiamenti awenuti e delle opere state eseguite nella R. Città di Pavia sul fine del Secolo XVIII, e nel principio del XIX infino all'Anno MDCCCXXX. Pavia: Stamperia Fusi e Comp.

Halbwachs Maurice (200I). La memoria collettiva. Milano: Unicopli.

Lucenti Simone (20 I 8). II recupero di edifici ex ecclesiastici a uso universitario a Pavia.The refurbishment of former ecclesiastical buildings for university use in Pavia. In Colloquiate 2018. Convegno Ar.Tec., Cagliari 12-14 settembre 2018. Monfalcone (Gorizia): EdicomEdizioni, pp. 108-121.

Mantovani Dario (20 I2). II lungo cammino dei mercanti di sapienza. Le origini dell'Università di Pavia nella storiografia dal XIV al XX secolo. In Mantovani (a cura di). Almum Studium Papiense. Storia dell'Università di Pavia. Dalle origini all'età spagnola. Tomo I. Origini e fondazione dello Studium generale. Volume I. Milano: Cisalpino Istituto Editoriale Universitario, pp. 29-82.

Morandotti Marco, Besana Daniela, Zamperini Emanuele, et al. (20|4). La gestione sostenibile del patrimonio immobiliare tra riuso e valorizzazione. In Della Torre (a cura di). La strategia della Conservazione programmata, dalla progettazione delle attivita alla valutazione degli impatti. Proceedings if the International Conference Preventive and Planned Conservation, MonzaMantova 5-9 maggio 2014. Milano: Nardini Editore, pp. 863-874.

Morandotti Marco, Parrinello Sandro, Picchio Francesca et al. (2019). L'Università di Pavia. I cortili e gli ambienti monumentali. Un progetto di documentazione digitale e sviluppo di sistemi di gestione per la manutenzione programmata. In ReUSO, VII Convegno Internazionale sulla documentazione, conservazione recupero del patrimonio architettonico e sulla tutela paesaggistica. Matera 23-26 ottobre 2019. Roma: Gangemi editore, pp. I31-140.

Pavesi Pietro (I897) La strada delle catene. Pavia: Stabilimento tipografico succ. Bizzoni (Ristampa: Pavia: Stamperia Fusi e Comp. 1955).

Parrinello Sandro (20I5). Rilevare, disegnare e organizzare la città digitale. In AAVV. Drawing \& City, Disegno \& Città. Cultura Scienza Arte Informazione, $37^{\circ}$ Convegno Internazionale dei Docenti della Rappresentazione. 17 - 19 sttembre 20 I5. Roma: Gangemi Editore, 261-270.

Parrinello Sandro, Picchio Francesca, Bercigli Monica (20I6). La "migrazione" della realtà in scenari virtuali: banche dati e sistemi di documentazione per la musealizzazione di ambienti complessi. In Disegnarecon, Vol. 9 n. I7, pp. I4.I - |4.8.

Parrinello Sandro, Picchio Francesca, Becherini Pietro et al. (20I8). The drawn landscape in 3D databases: the management of complexity and representation in the historical city. In 7th Annual International Conference on urban Studies \& Planning. ATINER'S CONFERENCE PAPER SERIES. Athens: Greece by the Athens Institute for Education and Research, pp. I-26.

Parrinello Sandro, Picchio Francesca, De Marco Raffaella et al. (2019). Documenting the cultural heritage routes. The creation of informative models of historical russian churches on upper kama region. In The International Archives of the Photogrammetry, Remote Sensing and Spatial Information Sciences, Volume XLII-2/W I 5, 2019. 27th CIPA International Symposium "Documenting the past for a better future", I -5 September 20 19, Ávila, Spain: <https://doi.org/ I 0.5 I 94/isprs-archives-XLII-2-W I 5-887-20 I 9>

Radice Flavia (2014). Reuse of deconsacrated churches: the case of Pavia. In XXXVII Curset jornades internacionals sobre la intervenció en el patrimoni arquitectònic. "Patrimoni sacre: permanent innovació". Barcelona: AADIPA.

\section{Author}

Francesca Picchio, Università degli Studi di Pavia, francesca.picchio@unipv.it

Elisabetta Doria, Università degli Studi di Pavia, elisabetta.doria@unipv.it

Alessia Miceli, Università degli Studi di Pavia, alessia.miceli@unipv.it

To cite this chapter. Picchio Francesca, Doria Elisabetta, Miceli Alessia (2020). Definizione di banche dati e procedure per la valorizzazione del Palazzo Centrale dell'Università di Pavia/Definition of databases and procedures for the valorization of Central Palace of University of Pavia. In Arena A., Arena M., Brandolino R.G., Colistra D., Ginex G., Mediati D., Nucifora S., Raffa P. (a cura di). Connettere. Un disegno per annodare e tessere Atti del $42^{\circ}$ Convegno Internazionale dei Docenti delle Discipline della Rappresentazione/Connecting. Drawing for weaving relationships. Proceedings of the 42th International Conference of Representation Disciplines Teachers. Milano: FrancoAngeli, pp. 2582-2603. 